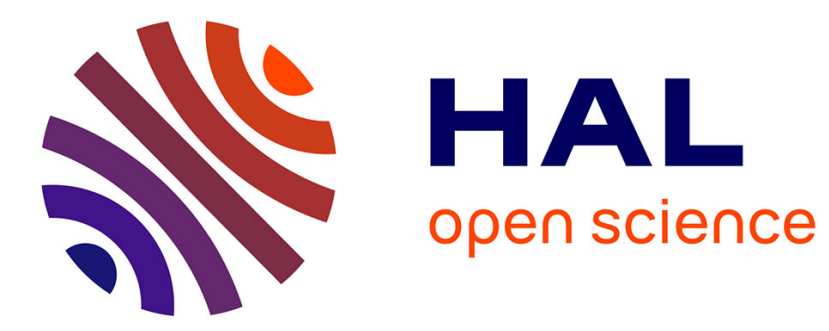

\title{
Vertex Approximate Gradient Scheme for Hybrid Dimensional Two-Phase Darcy Flows in Fractured Porous Media
}

Konstantin Brenner, Mayya Groza, Cindy Guichard, Roland Masson

\section{- To cite this version:}

Konstantin Brenner, Mayya Groza, Cindy Guichard, Roland Masson. Vertex Approximate Gradient Scheme for Hybrid Dimensional Two-Phase Darcy Flows in Fractured Porous Media. ESAIM: Mathematical Modelling and Numerical Analysis, 2014, pp.49 2 (2015) 303-330. 10.1051/m2an/2014034 . hal-00910939v3

\section{HAL Id: hal-00910939 \\ https://hal.science/hal-00910939v3}

Submitted on 5 Feb 2015

HAL is a multi-disciplinary open access archive for the deposit and dissemination of scientific research documents, whether they are published or not. The documents may come from teaching and research institutions in France or abroad, or from public or private research centers.
L'archive ouverte pluridisciplinaire HAL, est destinée au dépôt et à la diffusion de documents scientifiques de niveau recherche, publiés ou non, émanant des établissements d'enseignement et de recherche français ou étrangers, des laboratoires publics ou privés. 


\title{
Vertex Approximate Gradient Scheme for Hybrid Dimensional Two-Phase Darcy Flows in Fractured Porous Media
}

\author{
K. Brenner*, M. Groza ${ }^{\dagger}$ C. Guichard; R. Masson ${ }^{\S}$
}

December 21, 2014

\begin{abstract}
This paper presents a finite volume discretization of two-phase Darcy flows in discrete fracture networks taking into account the mass exchange between the matrix and the fracture. We consider the asymptotic model for which the fractures are represented as interfaces of codimension one immersed in the matrix domain, leading to the so called hybrid dimensional Darcy flow model. The pressures at the interfaces between the matrix and the fracture network are continuous corresponding to a ratio between the normal permeability of the fracture and the width of the fracture assumed to be large compared with the ratio between the permeability of the matrix and the size of the domain. The discretization is an extension of the Vertex Approximate Gradient (VAG) scheme to the case of hybrid dimensional Darcy flow models. Compared with Control Volume Finite Element (CVFE) approaches, the VAG scheme has the advantage to avoid the mixing of the fracture and matrix rocktypes at the interfaces between the matrix and the fractures, while keeping the low cost of a nodal discretization on unstructured meshes. The convergence of the scheme is proved under the assumption that the relative permeabilities are bounded from below by a strictly positive constant. This assumption is needed in the convergence proof in order to take into account discontinuous capillary pressures in particular at the matrix fracture interfaces. The efficiency of our approach compared with CVFE discretizations is shown on two numerical examples of fracture networks in $2 \mathrm{D}$ and $3 \mathrm{D}$.
\end{abstract}

\section{Introduction}

This article deals with the discretization of two-phase Darcy flows in fractured porous media for which the fractures are modelized as interfaces of codimension one. In this framework, the $d-1$ dimensional flow in the fractures is coupled with the $d$ dimensional flow in the matrix leading to the so called, hybrid dimensional Darcy flow model. We focus on the particular case where the pressure is continuous at the interfaces between the fractures and the matrix domain. This type of hybrid dimensional Darcy flow model has been introduced in [1] for single phase Darcy flows and in [29], [28], [21] for two-phase Darcy flows. It corresponds physically to pervious fractures for which the ratio of the transversal permeability of the fracture to the width of the fracture is large compared with the ratio of the permeability of the matrix to the size of the domain. Note that it does not cover the case of fractures acting as barriers for which the pressure is discontinuous at the matrix fracture interfaces (see [18], [26], [3] for discontinuous pressure models).

It is also assumed in our model that the pressure is continuous at the fracture intersections. It corresponds to a high ratio assumption between the permeability at the fracture intersections and the

\footnotetext{
${ }^{*}$ Laboratoire de Mathématiques J.A. Dieudonné, UMR 7351 CNRS, University Nice Sophia Antipolis, and team COFFEE, INRIA Sophia Antipolis Méditerranée, Parc Valrose 06108 Nice Cedex 02, France, konstantin.brenner@unice.fr

${ }^{\dagger}$ Laboratoire de Mathématiques J.A. Dieudonné, UMR 7351 CNRS, University Nice Sophia Antipolis, and team COFFEE, INRIA Sophia Antipolis Méditerranée, Parc Valrose 06108 Nice Cedex 02, France, mayya.groza@unice.fr

${ }^{\ddagger}$ Sorbonne Universités, UPMC Univ Paris 06, UMR 7598, CNRS, Laboratoire Jacques-Louis Lions, F-75005, Paris, and INRIA, ANGE project-team, Rocquencourt - B.P. 105, F78153 Le Chesnay cedex, and CEREMA, ANGE projectteam, 134 rue de Beauvais, F-60280 Margny-Lès-Compiègne, France, cindy.guichard@ann.jussieu.fr

${ }^{\S}$ Laboratoire de Mathématiques J.A. Dieudonné, UMR 7351 CNRS, University Nice Sophia Antipolis, and team COFFEE, INRIA Sophia Antipolis Méditerranée, Parc Valrose 06108 Nice Cedex 02, France, roland.masson@unice.fr
} 
width of the fracture compared with the ratio between the tangential permeability of each fracture and its lengh. We refer to [19] for a more general reduced model taking into account discontinuous pressures at fracture intersections in dimension $d=2$.

In the framework of two-phase Darcy flows in fractured porous media, high contrasts of capillary pressures are expected in particular between the matrix and the fractures. Hence, it is crucial to take into account in the model formulation the saturation jumps at the matrix fracture interfaces. We refer to [11], [8], [9] for mathematical formulations taking into account discontinuous capillary pressures. In the present work, we employ the phase pressures formulation (see e.g. [17]) which is an ideal framework to capture the saturation jump condition at the interface between different rocktypes without introducing any additional unknowns at these interfaces.

The discretization of the hybrid dimensional Darcy flow model with continuous pressures has been the object of several works. In [24] a cell-centred Finite Volume scheme using a Two Point Flux Approximation (TPFA) is proposed assuming the orthogonality of the mesh and isotropic permeability fields. Cell-centred Finite Volume schemes can be extended to general meshes and anisotropic permeability fields using MultiPoint Flux Approximations (MPFA) following the ideas introduced in [31] for discontinuous pressure models. Nevertheless, MPFA schemes can lack robustness on distorted meshes and large anisotropies due to the non symmetry of the discretization. They are also very expensive compared with nodal discretizations on tetrahedral meshes. In [1], a Mixed Finite Element (MFE) method is proposed for single Darcy flows. It is extended to two-phase flows in [21] in an IMPES framework using a Mixed Hybrid Finite Element (MHFE) discretization for the pressure equation and a Discontinuous Galerkin discretization of the saturation equation. These approaches are adapted to general meshes and anisotropy but require as many degrees of freedom as faces. Control Volume Finite Element Methods (CVFE) [29], [28] have the advantage to use only nodal unknowns leading to much fewer degrees of freedom than MPFA and MHFE schemes on tetrahedral meshes. On the other hand, at the matrix fracture interfaces, the control volumes have the drawback to be shared between the matrix and the fractures. It results that a strong refinement of the mesh is needed at these interfaces in the case of large contrasts between the permeabilities of the matrix and of the fractures.

In this article we extend the Vertex Approximate Gradient (VAG) scheme to the framework of hybrid dimensional two-phase Darcy flows. The VAG scheme has been introduced for the discretization of multiphase Darcy flows in [14] for immiscible flows and in [15] for compositional models. The VAG scheme basically uses nodal unknowns like the CVFE method but it also keep the cell-centred unknowns, which are eliminated at the linear algebra level without any fill-in. Compared with CVFE methods, it has the advantage to provide a large flexibility in the choice of the control volumes in order to avoid mixing rocks with highly contrasted absolute permeabilities in a single control volume. Compared with cell centred schemes such as the MPFA O scheme, it exhibits a better robustness regarding anisotropy [20] and a much cheaper cost on tetrahedral meshes [15]. In [17] it is further adapted to take into account discontinuous capillary pressures using a phase pressures formulation in order to capture the saturation jumps at different rocktype interfaces. It is this latter approach that is extended, in this article, to the case of hybrid dimensional two-phase Darcy flows in fractured porous media.

The first convergence result for a finite volume discretization of single media two-phase Darcy flow models has been obtained for cell-centred TPFA schemes on admissible meshes in [27] and [12]. In [12] the convergence is obtained for the usual phase pressures and saturations formulation using a phase by phase upwinding of the mobilities. In [27] the convergence is obtained for the global pressure formulation introduced in [10] (see also [4]). This latter convergence result has been recently extended in [6] to the case of the VAG discretization. The convergence is shown to hold whatever the choice of the volumes at the nodal unknowns. In the case of two-phase Darcy flows with discontinuous capillary pressure, the convergence of a TPFA type discretization is obtained in [7]. The extension to general meshes is done in [17] assuming the non degeneracy of the relative permeabilities in the framework of 
gradient scheme discretizations introduced in [13].

To our knowledge, there is not yet a proof of convergence for hybrid dimensional two-phase Darcy flows. In this paper, we propose to extend the results obtained in [17] in the particular case of the VAG scheme to the hybrid dimensional Darcy flow model. The convergence of the VAG scheme to a weak solution is obtained assuming that the relative permeabilities of both phases are bounded from below by a strictly positive constant, and assuming that the family of meshes is shape regular.

The outline of the article is the following. In section 1 , the hybrid dimensional model is introduced and its discretization using the VAG scheme is described in section 2. The convergence of the scheme is obtained in section 3 assuming the non degeneracy of the relative permeabilities and whatever the choice of the volumes at the nodal unknowns. The numerical tests presented in section 4 exhibit the efficiency of our approach in both 2D and 3D cases. In particular, for large contrasts of permeability between the matrix and the fractures, it is shown that the VAG scheme provides a much more accurate solution than CVFE approaches for a similar cost.

\section{Hybrid dimensional Two-Phase Darcy Flow Model in Fractured Porous Media}

\subsection{Hybrid Dimensional Model in Fractured Porous Media}

Let $\Omega$ denote a bounded domain of $\mathbb{R}^{d}, d=2,3$ assumed to be polyhedral for $d=3$ and polygonal for $d=2$. To fix ideas the dimension will be fixed to $d=3$ when it needs to be specified, for instance in the naming of the geometrical objects or for the space discretization in the next section. The adaptations to the case $d=2$ are straightforward.

We consider the asymptotic model introduced in [1] where fractures are represented as interfaces of codimension 1. Let $\bar{\Gamma}=\bigcup_{i \in I} \bar{\Gamma}_{i}$ and its interior $\Gamma=\bar{\Gamma} \backslash \partial \bar{\Gamma}$ denote the network of fractures $\Gamma_{i} \subset \Omega$, $i \in I$, such that each $\Gamma_{i}$ is a planar polygonal simply connected open domain included in a plane $\mathcal{P}_{i}$ of $\mathbb{R}^{d}$. It is assumed that the angles of $\Gamma_{i}$ are strictly lower than $2 \pi$ and that $\Gamma_{i} \cap \bar{\Gamma}_{j}=\emptyset$ for all $i \neq j$. For all $i \in I$, let us set $\Sigma_{i}=\partial \Gamma_{i}, \Sigma_{i, j}=\Sigma_{i} \cap \Sigma_{j}, j \in I, \Sigma_{i, 0}=\Sigma_{i} \cap \partial \Omega, \Sigma_{i, N}=\Sigma_{i} \backslash\left(\bigcup_{j \in I} \Sigma_{i, j} \cup \Sigma_{i, 0}\right)$, and $\Sigma=\bigcup_{(i, j) \in I \times I, i \neq j} \Sigma_{i, j}$. It is assumed that $\Sigma_{i, 0}=\bar{\Gamma}_{i} \cap \partial \Omega$.

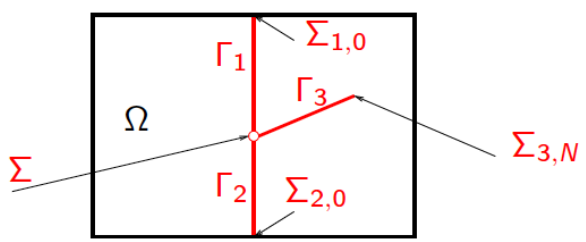

Figure 1: Example of a 2D domain $\Omega$ and 3 intersecting fractures $\Gamma_{i}, i=1,2,3$.

We will denote by $d \tau(\mathbf{x})$ the $d-1$ dimensional Lebesgue measure on $\Gamma$. On the fracture network $\Gamma$, we define the function space $L^{2}(\Gamma)=\left\{v=\left(v_{i}\right)_{i \in I}, v_{i} \in L^{2}\left(\Gamma_{i}\right), i \in I\right\}$, endowed with the norm $\|v\|_{L^{2}(\Gamma)}^{2}=\sum_{i \in I}\left\|v_{i}\right\|_{L^{2}\left(\Gamma_{i}\right)}^{2}$ and its subspace $H^{1}(\Gamma)$ consisting of functions $v=\left(v_{i}\right)_{i \in I}$ such that $v_{i} \in H^{1}\left(\Gamma_{i}\right), i \in I$ with continuous traces at the fracture intersections. The space $H^{1}(\Gamma)$ is endowed with the norm $\|v\|_{H^{1}(\Gamma)}^{2}=\sum_{i \in I}\left\|v_{i}\right\|_{H^{1}\left(\Gamma_{i}\right)}^{2}$ and its subspace with vanishing traces on $\Sigma_{0}=\bigcup_{i \in I} \Sigma_{i, 0}$ is denoted by $H_{\Sigma_{0}}^{1}(\Gamma)$.

Let us also consider the trace operator $\gamma_{i}$ from $H^{1}(\Omega)$ to $L^{2}\left(\Gamma_{i}\right)$ as well as the trace operator $\gamma$ from $H^{1}(\Omega)$ to $L^{2}(\Gamma)$ such that $(\gamma v)_{i}=\gamma_{i}(v)$ for all $i \in I$.

On $\Omega$, the gradient operator from $H^{1}(\Omega)$ to $L^{2}(\Omega)^{d}$ is denoted by $\nabla$. On the fracture network $\Gamma$, 
the tangential gradient is denoted by $\nabla_{\tau}$ from $H^{1}(\Gamma)$ to $L^{2}(\Gamma)^{d-1}$ and such that

$$
\nabla_{\tau} v=\left(\nabla_{\tau_{i}} v_{i}\right)_{i \in I},
$$

where, for each $i \in I$, the tangential gradient $\nabla_{\tau_{i}}$ is defined from $H^{1}\left(\Gamma_{i}\right)$ to $L^{2}\left(\Gamma_{i}\right)^{d-1}$ by fixing a reference Cartesian coordinate system of the plane $\mathcal{P}_{i}$ containing $\Gamma_{i}$. We also denote by $\operatorname{div}_{\tau_{i}}$ the divergence operator from $H_{\mathrm{div}}\left(\Gamma_{i}\right)$ to $L^{2}\left(\Gamma_{i}\right)$.

The function spaces used in the variational formulation of the hybrid dimensional Darcy flow model are defined by

$V=\left\{v \in H^{1}(\Omega)\right.$ such that $\left.\gamma v \in H^{1}(\Gamma)\right\}$, and its subspace $V^{0}=\left\{v \in H_{0}^{1}(\Omega)\right.$ such that $\left.\gamma v \in H_{\Sigma_{0}}^{1}(\Gamma)\right\}$.

From Poincaré inequality on $H_{0}^{1}(\Omega)$ and the continuity of the trace operator $\gamma$, we deduce the following inequality with a constant $C_{P}$ depending in particular on the number of fractures.

Proposition 1.1 There exists $C_{P}>0$ such that for all $v \in V^{0}$ one has

$$
\|v\|_{L^{2}(\Omega)}+\|\gamma v\|_{L^{2}(\Gamma)} \leq C_{P}\|\nabla v\|_{L^{2}(\Omega)^{d}} .
$$

Thus, the space $V^{0}$ is endowed with the following norm

$$
\|v\|_{V}=\left(\|\nabla v\|_{L^{2}(\Omega)^{d}}^{2}+\left\|\nabla_{\tau} \gamma v\right\|_{L^{2}(\Gamma)^{d-1}}^{2}\right)^{1 / 2},
$$

and the space $V$ with the norm $\|v\|_{V}^{2}=\|v\|_{V^{0}}^{2}+\|v\|_{L^{2}(\Omega)}^{2}$.

For all $i \in I$, we can define the two sides \pm of the fracture $\Gamma_{i}$ in $\Omega \backslash \bar{\Gamma}$ and the corresponding unit normal vectors $n_{i}^{ \pm}$at $\Gamma_{i}$ outward to the sides \pm . For all $\mathbf{q}_{m} \in H_{\operatorname{div}}(\Omega \backslash \bar{\Gamma})$, let $\mathbf{q}_{m}^{ \pm} \cdot n_{i}^{ \pm}$denote the two normal traces at the fracture $\Gamma_{i}$ in the sense of distributions. For all fractures $\Gamma_{i}, i \in I$, we also denote by $\mathbf{n}_{\Sigma_{i}}$ the unit vector normal to $\Sigma_{i}$ in the fracture plane $\mathcal{P}_{i}$ and outward to $\Gamma_{i}$.

\subsection{Two-Phase Darcy Flows in Phase Pressures Formulation}

In the framework of two-phase Darcy flows in fractured porous media high contrasts of capillary pressures are expected in particular at the interfaces between the matrix and the fractures. Hence, it is crucial to take into account in the model formulation the saturation jumps at these interfaces (see for example [8], [9] for the mathematical formulations of two-phase Darcy flows with discontinuous capillary pressures). Our choice focuses on the phase pressures formulation (see e.g. [17]) which is extended to the case of hybrid dimensional two-phase Darcy flows in a variational formulation framework. The main advantage of this formulation is to choose as primary unknowns the phase pressures which can be assumed to be continuous at the interfaces between different rocktypes while the jump of the saturation is captured using the inverse of the capillary pressure monotone graph for each rocktype. An alternative choice using a global pressure approach is presented in [23].

Let $u^{2}$ (resp. $u^{1}$ ) denote the wetting (resp. non wetting) phase pressure, $p=u^{1}-u^{2}$ the capillary pressure, and $p_{\text {ini }} \in V$ the initial capillary pressure. For the sake of simplicity in the convergence analysis, homogeneous Dirichlet boundary conditions are assumed for $u^{1}$ and $u^{2}$ at the boundary $\partial \Omega$, as well as at $\Sigma_{0}$ for $\gamma u^{1}$ and $\gamma u^{2}$. The gravity is also not taken into account in the model to simplify the analysis. The extension of the convergence proof to the case with gravity and with non homogeneous Dirichlet boundary conditions can be done easily following the same ideas as in [17].

Let us denote by $S_{m}^{1}(\mathbf{x}, p)$ (resp. $S_{f}^{1}(\mathbf{x}, p)$ ) the inverses of the monotone graph extension of the capillary pressure curves in the matrix domain $\Omega$ (resp. in the fracture network $\Gamma$ ), and let us set $S_{m}^{2}=1-S_{m}^{1}\left(\operatorname{resp} . S_{f}^{2}=1-S_{f}^{1}\right)$. 
In the matrix domain $\Omega$ (resp. in the fracture network $\Gamma$ ), let us denote by $k_{m}^{\alpha}\left(\mathbf{x}, S_{m}^{\alpha}\right.$ ) (resp. $\left.k_{f}^{\alpha}\left(\mathbf{x}, S_{f}^{\alpha}\right)\right), \alpha=1,2$, the phase mobilities, by $\phi_{m}(\mathbf{x})$ (resp. $\left.\phi_{f}(\mathbf{x})\right)$ the porosity, and by $\Lambda_{m}(\mathbf{x})$ (resp. $\left.\Lambda_{f}(\mathbf{x})\right)$ the permeability tensor. We also denote by $d_{f}(\mathbf{x}), \mathbf{x} \in \Gamma$ the width of the fractures, and by $d \tau_{f}(\mathbf{x})$ the weighted Lebesgue $d-1$ dimensional measure on $\Gamma$ defined by $d \tau_{f}(\mathbf{x})=d_{f}(\mathbf{x}) d \tau(\mathbf{x})$.

Let us define the function space of the fluxes denoted by $W(\Omega, \Gamma)$ and defined by

$W(\Omega, \Gamma)=\left\{\begin{array}{l}\mathbf{q}_{m} \in H_{\operatorname{div}}(\Omega \backslash \bar{\Gamma}), \mathbf{q}_{f}=\left(\mathbf{q}_{f, i}\right)_{i \in I} \in L^{2}(\Gamma)^{d-1} \text { such that } \\ r_{f, i}=\operatorname{div}_{\tau}\left(\mathbf{q}_{f, i}\right)-\mathbf{q}_{m}^{+} \cdot \mathbf{n}_{i}^{+}-\mathbf{q}_{m}^{-} \cdot \mathbf{n}_{i}^{-} \in L^{2}\left(\Gamma_{i}\right), i \in I, \text { and } \\ \int_{\Omega \backslash \bar{\Gamma}}\left(\mathbf{q}_{m} \cdot \nabla v+\operatorname{div}\left(\mathbf{q}_{m}\right) v\right) d \mathbf{x}+\sum_{i \in I} \int_{\Gamma_{i}}\left(\mathbf{q}_{f, i} \cdot \nabla_{\tau} \gamma v+r_{f, i} \gamma v\right) d \tau=0 \text { for all } v \in V^{0}\end{array}\right\}$,

Note that the last condition in this definition amounts to impose in a weak sense

(i) the sum to zero of the output normal fluxes at fracture intersections $\sum_{i \in I} \mathbf{q}_{f, i} \cdot \mathbf{n}_{\Sigma_{i}}=0$ on $\Sigma$, assuming that the intersection volume can be neglected,

(ii) a zero normal flux boundary condition at the immersed boundaries of the fractures $\mathbf{q}_{f, i} \cdot \mathbf{n}_{\Sigma_{i}}=0$ on $\Sigma_{i, N}, i \in I$, assuming that the width at the tip of the fracture is small compared to the lengh of the fracture.

With these notations, the strong formulation of our hybrid dimensional two phase Darcy flow model amounts to find formally $u^{\alpha} \in L^{2}\left(0, T ; V^{0}\right), \alpha=1,2$, and $\left(\mathbf{q}_{m}^{\alpha}, \mathbf{q}_{f}^{\alpha}\right) \in L^{2}(0, T ; W(\Omega, \Gamma))$ such that one has for $\alpha=1,2$ :

$$
\left\{\begin{aligned}
\phi_{m} \partial_{t}\left(S_{m}^{\alpha}(x, p)\right)+\operatorname{div}\left(\mathbf{q}_{m}^{\alpha}\right) & =h_{m}^{\alpha} & & \text { on } \Omega \backslash \bar{\Gamma}, \\
\phi_{f} d_{f} \partial_{t}\left(S_{f}^{\alpha}\left(x, \gamma_{i} p\right)\right)+\operatorname{div}_{\tau_{i}}\left(\mathbf{q}_{f, i}^{\alpha}\right)-\mathbf{q}_{m}^{\alpha,+} \cdot \mathbf{n}_{i}^{+}-\mathbf{q}_{m}^{\alpha,-} \cdot \mathbf{n}_{i}^{-} & =d_{f} h_{f}^{\alpha} & & \text { on } \Gamma_{i}, i \in I, \\
-k_{m}^{\alpha}\left(x, S_{m}^{\alpha}(x, p)\right) \Lambda_{m} \nabla u^{\alpha} & =\mathbf{q}_{m}^{\alpha} & & \text { on } \Omega \backslash \bar{\Gamma}, \\
-d_{f} k_{f}^{\alpha}\left(x, S_{f}^{\alpha}\left(x, \gamma_{i} p\right)\right) \Lambda_{f} \nabla_{\tau_{i}} \gamma_{i} u^{\alpha} & =\mathbf{q}_{f, i}^{\alpha} & & \text { on } \Gamma_{i}, i \in I, \\
\left.p\right|_{t=0} & =p_{\text {ini }}, & & \text { on } \Omega,
\end{aligned}\right.
$$

where the function $h_{m}^{\alpha}$ (resp. $h_{f}^{\alpha}$ ), $\alpha=1,2$ stands for the source terms in the matrix domain $\Omega$ (resp. in the fracture network $\Gamma$ ).

The corresponding weak formulation amounts to find $u^{1}, u^{2} \in L^{2}\left(0, T ; V^{0}\right)$ satisfying the following variational equalities for $\alpha=1,2$, and for all $\varphi \in C_{c}^{\infty}([0, T) \times \Omega)$ :

$$
\left\{\begin{array}{l}
\int_{0}^{T} \int_{\Omega}\left(-\phi_{m}(\mathbf{x}) S_{m}^{\alpha}(\mathbf{x}, p) \partial_{t} \varphi(\mathbf{x}, t)+k_{m}^{\alpha}\left(\mathbf{x}, S_{m}^{\alpha}(\mathbf{x}, p)\right) \Lambda_{m}(\mathbf{x}) \nabla u^{\alpha}(\mathbf{x}, t) \cdot \nabla \varphi(\mathbf{x}, t)\right) d \mathbf{x} d t \\
+\int_{0}^{T} \int_{\Gamma}-\phi_{f}(\mathbf{x}) S_{f}^{\alpha}(\mathbf{x}, \gamma p) \partial_{t} \gamma \varphi(\mathbf{x}, t) d \tau_{f}(\mathbf{x}) d t \\
+\int_{0}^{T} \int_{\Gamma} k_{f}^{\alpha}\left(\mathbf{x}, S_{f}^{\alpha}(\mathbf{x}, \gamma p)\right) \Lambda_{f}(\mathbf{x}) \nabla_{\tau} \gamma u^{\alpha}(\mathbf{x}, t) \cdot \nabla_{\tau} \gamma \varphi(\mathbf{x}, t) d \tau_{f}(\mathbf{x}) d t \\
-\int_{\Omega} \phi_{m}(\mathbf{x}) S_{m}^{\alpha}\left(\mathbf{x}, p_{\text {ini }}\right) \varphi(\mathbf{x}, 0) d \mathbf{x} d t-\int_{\Gamma} \phi_{f}(\mathbf{x}) S_{f}^{\alpha}\left(\mathbf{x}, \gamma p_{\text {ini }}\right) \varphi(\mathbf{x}, 0) d \tau_{f}(\mathbf{x}) d t \\
-\int_{0}^{T} \int_{\Omega} h_{m}^{\alpha}(\mathbf{x}, t) \varphi(\mathbf{x}, t) d \mathbf{x} d t-\int_{0}^{T} \int_{\Gamma} h_{f}^{\alpha}(\mathbf{x}, t) \gamma \varphi(\mathbf{x}, t) d \tau_{f}(\mathbf{x}) d t=0 .
\end{array}\right.
$$

As in [17], the following assumptions are made on the data:

(H1) $\phi_{m}$ is a measurable function from $\Omega$ to $\mathbb{R}$ with $\phi_{m}(\mathbf{x}) \in\left[\phi_{\min }, \phi_{\max }\right], \phi_{\max } \geq \phi_{\min }>0$. $\phi_{f}$ is a measurable function from $\Gamma$ to $\mathbb{R}$ with $\phi_{f}(\mathbf{x}) \in\left[\phi_{\min }, \phi_{\max }\right] . d_{f}$ is a measurable function from $\Gamma$ to $\mathbb{R}$ with $d_{f}(\mathbf{x}) \in\left[d_{\min }, d_{\max }\right], d_{\max } \geq d_{\min }>0$.

(H2) $\Lambda_{m}$ is a measurable function from $\Omega$ to $\mathcal{M}_{d}(\mathbb{R})$ (where $\mathcal{M}_{r}(\mathbb{R})$ denotes the set of $r \times r$ matrices with real coefficients) such that for a.e. $\mathbf{x} \in \Omega, \Lambda_{m}(\mathbf{x})$ is symmetric and the set of its eigenvalues 
is included in $\left[\lambda_{\min }, \lambda_{\max }\right]$ with $0<\lambda_{\min } \leq \lambda_{\max } . \Lambda_{f}$ is a measurable function from $\Gamma$ to $\mathcal{M}_{d-1}(\mathbb{R})$ such that for a.e. $\mathbf{x} \in \Gamma, \Lambda_{f}(\mathbf{x})$ is symmetric and the set of its eigenvalues is included in $\left[\lambda_{\min }, \lambda_{\max }\right]$.

(H3) $S_{m}^{1}(\mathbf{x}, p) \in[0,1]$ for all $(\mathbf{x}, p) \in \Omega \times \mathbb{R}$ with $S_{m}^{1}(\mathbf{x}, p)=S_{m, j}^{1}(p)$ for a.e. $\mathbf{x} \in \Omega_{j}$ and all $p \in \mathbb{R}$, where $S_{m, j}^{1}$ is a non decreasing Lipschitz continuous function with constant $L_{S}$ and $\left(\Omega_{j}\right)_{j \in J_{m}}$ is a finite family of disjoint connected polyhedral open sets such that $\bigcup_{j \in J_{m}} \overline{\Omega_{j}}=\bar{\Omega} . S_{f}^{1}(\mathbf{x}, p) \in[0,1]$ for all $(\mathbf{x}, p) \in \Gamma \times \mathbb{R}$ with $S_{f}^{1}(\mathbf{x}, p)=S_{f, j}^{1}(p)$ for a.e. $\mathbf{x} \in \Upsilon_{j}$ and all $p \in \mathbb{R}$, where $S_{f, j}^{1}$ is a non decreasing Lipschitz continuous function with constant $L_{S}$ and $\left(\Upsilon_{j}\right)_{j \in J_{f}}$ is a finite family of disjoint connected polygonal open sets such that $\bigcup_{j \in J_{f}} \overline{\Upsilon_{j}}=\bar{\Gamma}$.

(H4) $k_{m}^{\alpha}(\mathbf{x}, s)\left(\operatorname{resp} . k_{f}^{\alpha}(\mathbf{x}, s)\right) \in\left[k_{\min }, k_{\max }\right]$ for $(\mathbf{x}, s) \in \Omega \times[0,1](\operatorname{resp} .(\mathbf{x}, s) \in \Gamma \times[0,1]), k_{\max } \geq$ $k_{\min }>0$ and $k_{m}^{\alpha}(\cdot, s)\left(\operatorname{resp} . k_{f}^{\alpha}(\cdot, s)\right)$ measurable, $k_{m}^{\alpha}(\mathbf{x}, \cdot)\left(\operatorname{resp} . k_{f}^{\alpha}(\mathbf{x}, \cdot)\right)$ continuous, $\alpha=1,2$.

(H5) $p_{\text {ini }} \in V, h_{m}^{\alpha} \in L^{2}(\Omega \times(0, T)), h_{f}^{\alpha} \in L^{2}(\Gamma \times(0, T)), \alpha=1,2$.

Assumptions (H1-H5) are quite general, except for $k_{\min }>0$ of hypothesis $\mathrm{H} 4$. This assumption is needed in the mathematical part of this paper. Remark that it is not needed in the implementation of the numerical scheme and will be dropped in the numerical section. The influence of this parameter has already been studied numerically in [17]. The hypothesis (H3) that the functions $S_{m}^{1}(\mathbf{x}, p)$ and $S_{f}^{1}(\mathbf{x}, p)$ are defined by given functions in a partition of the domain is classical and the index $j$ corresponds to the so called geological rocktypes.

\section{Vertex Approximate Gradient Discretization}

In the spirit of [13], we consider generalised polyhedral meshes of $\Omega$. Let $\mathcal{M}$ be the set of cells that are disjoint open polyhedral subsets of $\Omega$ such that $\bigcup_{K \in \mathcal{M}} \bar{K}=\bar{\Omega}$. For all $K \in \mathcal{M}, \mathbf{x}_{K}$ denotes the so-called "centre" of the cell $K$ under the assumption that $K$ is star-shaped with respect to $\mathbf{x}_{K}$. We then denote by $\mathcal{F}_{K}$ the set of interfaces of non zero $d-1$ dimensional measure among the interior faces $\bar{K} \cap \bar{L}, L \in \mathcal{M}$, and the boundary interface $\bar{K} \cap \partial \Omega$, which possibly splits in several boundary faces. Let us denote by

$$
\mathcal{F}=\bigcup_{K \in \mathcal{M}} \mathcal{F}_{K}
$$

the set of all faces of the mesh. Remark that the faces are not assumed to be planar, hence the term "generalised polyhedral mesh". For $\sigma \in \mathcal{F}$, let $\mathcal{E}_{\sigma}$ be the set of interfaces of non zero $d-2$ dimensional measure among the interfaces $\bar{\sigma} \cap \bar{\sigma}^{\prime}, \sigma^{\prime} \in \mathcal{F}$. Then, we denote by

$$
\mathcal{E}=\bigcup_{\sigma \in \mathcal{F}} \mathcal{E}_{\sigma}
$$

the set of all edges of the mesh. Let $\mathcal{V}_{\sigma}=\bigcup_{e, e^{\prime} \in \mathcal{E}_{\sigma}, e \neq e^{\prime}}\left(e \cap e^{\prime}\right)$ be the set of vertices of $\sigma$. For each $K \in \mathcal{M}$ we define $\mathcal{V}_{K}=\bigcup_{\sigma \in \mathcal{F}_{K}} \mathcal{V}_{\sigma}$, and we also denote by

$$
\mathcal{V}=\bigcup_{K \in \mathcal{M}} \mathcal{V}_{K}
$$

the set of all vertices of the mesh. It is then assumed that for each face $\sigma \in \mathcal{F}$, there exists a so-called "centre" of the face $\mathbf{x}_{\sigma} \in \sigma \backslash \bigcup_{e \in \mathcal{E}_{\sigma}} e$ such that $\mathbf{x}_{\sigma}=\sum_{\mathbf{s} \in \mathcal{V}_{\sigma}} \beta_{\sigma, \mathbf{s}} \mathbf{x}_{\mathbf{s}}$, with $\sum_{\mathbf{s} \in \mathcal{V}_{\sigma}} \beta_{\sigma, \mathbf{s}}=1$, and $\beta_{\sigma, \mathbf{s}} \geq 0$ for all $\mathbf{s} \in \mathcal{V}_{\sigma}$; moreover the face $\sigma$ is assumed to be defined by the union of the triangles $T_{\sigma, e}$ defined by the face centre $\mathbf{x}_{\sigma}$ and each edge $e \in \mathcal{E}_{\sigma}$.

The mesh is also supposed to be conforming w.r.t. the fracture network $\Gamma$ in the sense that for all $i \in I$ there exist the subsets $\mathcal{F}_{\Gamma_{i}}$ of $\mathcal{F}$ such that $\bar{\Gamma}_{i}=\bigcup_{\sigma \in \mathcal{F}_{\Gamma_{i}}} \bar{\sigma}$. We will denote by $\mathcal{F}_{\Gamma}$ the set of fracture faces $\bigcup_{i \in I} \mathcal{F}_{\Gamma_{i}}$. This geometrical discretization of $\Omega$ and $\Gamma$ is denoted in the following by $\mathcal{D}$. 
The VAG discretization has been introduced in [13] for diffusive problems on heterogeneous anisotropic media. Its extension to the hybrid dimensional Darcy model is based on the following vector space of degrees of freedom:

$$
X_{\mathcal{D}}=\left\{v_{K}, v_{\mathbf{s}}, v_{\sigma} \in \mathbb{R}, K \in \mathcal{M}, \mathbf{s} \in \mathcal{V}, \sigma \in \mathcal{F}_{\Gamma}\right\},
$$

and its subspace with homogeneous Dirichlet boundary conditions on $\partial \Omega$ :

$$
X_{\mathcal{D}}^{0}=\left\{v \in X_{\mathcal{D}} \mid v_{\mathbf{s}}=0 \text { for } \mathbf{s} \in \mathcal{V}_{\text {ext }}\right\} .
$$

where $\mathcal{V}_{\text {ext }}=\mathcal{V} \cap \partial \Omega$ denotes the set of boundary vertices, and $\mathcal{V}_{\text {int }}=\mathcal{V} \backslash \partial \Omega$ denotes the set of interior vertices.

A finite element discretization of $V$ is built using a tetrahedral sub-mesh of $\mathcal{M}$ and a second order interpolation at the face centres $\mathbf{x}_{\sigma}, \sigma \in \mathcal{F} \backslash \mathcal{F}_{\Gamma}$ defined by the operator $I_{\sigma}: X_{\mathcal{D}} \rightarrow \mathbb{R}$ such that

$$
I_{\sigma}(v)=\sum_{\mathbf{s} \in \mathcal{V}_{\sigma}} \beta_{\sigma, \mathbf{s}} v_{\mathbf{s}}
$$

The tetrahedral sub-mesh is defined by $\mathcal{T}=\left\{T_{K, \sigma, e}, e \in \mathcal{E}_{\sigma}, \sigma \in \mathcal{F}_{K}, K \in \mathcal{M}\right\}$ where $T_{K, \sigma, e}$ is the tetrahedron joining the cell centre $\mathbf{x}_{K}$ to the triangle $T_{\sigma, e}$ (see Figure 2 for examples of such tetrahedra).

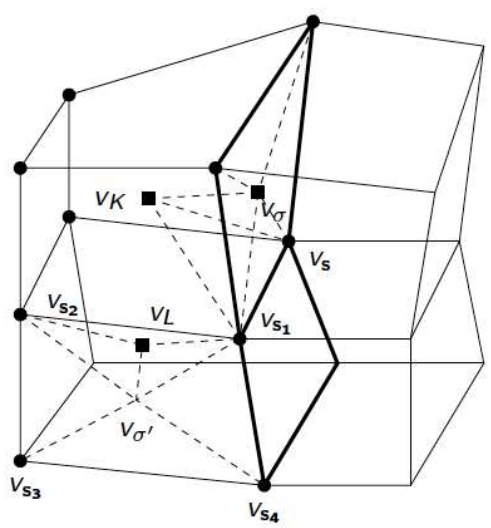

Figure 2: Degrees of freedom of the VAG scheme: cell unknowns $v_{K}, v_{L}$, fracture face unknown $v_{\sigma}$, and node unknowns $v_{\mathbf{s}}, v_{\mathbf{s}_{1}}, v_{\mathbf{s}_{2}}, v_{\mathbf{s}_{3}}, v_{\mathbf{s}_{4}}$. The fracture faces of $\mathcal{F}_{\Gamma}$ are in bold. The value of $v_{\sigma^{\prime}}$ is obtained by interpolation of the node unknowns $v_{\mathbf{s}_{1}}, v_{\mathbf{s}_{2}}, v_{\mathbf{s}_{3}}, v_{\mathbf{s}_{4}}$ of the face $\sigma^{\prime} \in \mathcal{F} \backslash \mathcal{F}_{\Gamma}$ while $v_{\sigma}$ is kept as an unknown for $\sigma \in \mathcal{F}_{\Gamma}$.

For a given $v \in X_{\mathcal{D}}$, we define the function $\pi_{\mathcal{T}} v \in V$ as the continuous piecewise affine function on each tetrahedron of $\mathcal{T}$ such that $\pi_{\mathcal{T}} v\left(\mathbf{x}_{K}\right)=v_{K}, \pi_{\mathcal{T}} v(\mathbf{s})=v_{\mathbf{s}}, \pi_{\mathcal{T}} v\left(\mathbf{x}_{\sigma}\right)=v_{\sigma}$, and $\pi_{\mathcal{T}} v\left(\mathbf{x}_{\sigma^{\prime}}\right)=I_{\sigma^{\prime}}(v)$ for all $K \in \mathcal{M}, \mathbf{s} \in \mathcal{V}, \sigma \in \mathcal{F}_{\Gamma}$, and $\sigma^{\prime} \in \mathcal{F} \backslash \mathcal{F}_{\Gamma}$. We define the conforming approximation of the space $V$ by

$$
V_{\mathcal{T}}=\left\{\pi_{\mathcal{T}} v, v \in X_{\mathcal{D}}\right\} \subset V
$$

and its subspace with homogeneous Dirichlet boundary conditions on $\partial \Omega$ :

$$
V_{\mathcal{T}}^{0}=\left\{\pi_{\mathcal{T}} v, v \in X_{\mathcal{D}}^{0}\right\}=V_{\mathcal{T}} \cap V^{0} .
$$

The nodal basis of this finite element discretization will be denoted by $\eta_{K}, \eta_{\mathbf{s}}, \eta_{\sigma}$, for $K \in \mathcal{M}, \mathbf{s} \in \mathcal{V}$, $\sigma \in \mathcal{F}_{\Gamma}$.

Discrete gradient operators are obtained from this finite element discretization of $V$, defining, for the matrix domain, the operator

$$
\nabla_{\mathcal{D}_{m}}: X_{\mathcal{D}} \rightarrow L^{2}(\Omega)^{d} \text { such that } \nabla_{\mathcal{D}_{m}} v=\nabla \pi_{\mathcal{T}} v,
$$


and, for the fracture network, the operator

$$
\nabla_{\mathcal{D}_{f}}: X_{\mathcal{D}} \rightarrow L^{2}(\Gamma)^{d-1} \text { such that } \nabla_{\mathcal{D}_{f}} v=\nabla_{\tau} \gamma \pi_{\mathcal{T}} v \text {. }
$$

In addition to the conforming finite element discretization, the VAG discretization uses two non conforming piecewise constant reconstructions of functions from $X_{\mathcal{D}}$ into respectively $L^{2}(\Omega)$ and $L^{2}(\Gamma)$ based on a partition of the cells and of the fracture faces. These partitions are respectively denoted, for all $K \in \mathcal{M}$, by

$$
K=\omega_{K} \bigcup\left(\bigcup_{\mathbf{s} \in \mathcal{V}_{K} \cap \mathcal{V}_{i n t}} \omega_{K, \mathbf{s}}\right) \bigcup\left(\bigcup_{\sigma \in \mathcal{F}_{K} \cap \mathcal{F}_{\Gamma}} \omega_{K, \sigma}\right),
$$

and, for all $\sigma \in \mathcal{F}_{\Gamma}$, by

$$
\sigma=\Sigma_{\sigma} \bigcup\left(\bigcup_{\mathbf{s} \in \mathcal{V}_{\sigma} \cap \mathcal{V}_{i n t}} \Sigma_{\sigma, \mathbf{s}}\right)
$$

Then, the function reconstruction operators are defined by

$$
\pi_{\mathcal{D}_{m}} v(\mathbf{x})= \begin{cases}v_{K} & \text { for all } \mathbf{x} \in \omega_{K}, K \in \mathcal{M}, \\ v_{\mathbf{s}} & \text { for all } \mathbf{x} \in \omega_{K, \mathbf{s}}, \mathbf{s} \in \mathcal{V}_{K} \cap \mathcal{V}_{i n t}, K \in \mathcal{M}, \\ v_{\sigma} & \text { for all } \mathbf{x} \in \omega_{K, \sigma}, \quad \sigma \in \mathcal{F}_{K} \cap \mathcal{F}_{\Gamma}, K \in \mathcal{M},\end{cases}
$$

and

$$
\pi_{\mathcal{D}_{f}} v(\mathbf{x})= \begin{cases}v_{\sigma} & \text { for all } \mathbf{x} \in \Sigma_{\sigma}, \sigma \in \mathcal{F}_{\Gamma} \\ v_{\mathbf{s}} & \text { for all } \mathbf{x} \in \Sigma_{\sigma, \mathbf{s}}, \mathbf{s} \in \mathcal{V}_{\sigma} \cap \mathcal{V}_{i n t}, \sigma \in \mathcal{F}_{\Gamma} .\end{cases}
$$

It is important to notice that in the particular case when the space discretization is conforming with respect to the sets $\left(\Omega_{j}\right)_{j \in J_{m}},\left(\Gamma_{j}\right)_{j \in J_{f}}$ and when the source term $h_{m}^{\alpha}$ (resp. $h_{f}^{\alpha}$ ) is a cellwise (resp. facewise) constant function, the implementation of the VAG scheme does not require to build these partitions. In that case, it is sufficient to define the matrix volume fractions

$$
\alpha_{K, \mathbf{s}}=\frac{\int_{\omega_{K, \mathbf{s}}} d \mathbf{x}}{\int_{K} d \mathbf{x}}, \mathbf{s} \in \mathcal{V}_{K} \cap \mathcal{V}_{i n t}, K \in \mathcal{M}, \alpha_{K, \sigma}=\frac{\int_{\omega_{K, \sigma}} d \mathbf{x}}{\int_{K} d \mathbf{x}}, \sigma \in \mathcal{F}_{K} \cap \mathcal{F}_{\Gamma}, K \in \mathcal{M}
$$

constrained to satisfy $\alpha_{K, \mathbf{s}} \geq 0, \alpha_{K, \sigma} \geq 0$, and $\sum_{\mathbf{s} \in \mathcal{V}_{K} \cap \mathcal{V}_{i n t}} \alpha_{K, \mathbf{s}}+\sum_{\sigma \in \mathcal{F}_{K} \cap \mathcal{F}_{\Gamma}} \alpha_{K, \sigma} \leq 1$, as well as the fracture volume fractions

$$
\alpha_{\sigma, \mathbf{s}}=\frac{\int_{\Sigma_{\sigma, \mathbf{s}}} d \tau_{f}(\mathbf{x})}{\int_{\sigma} d \tau_{f}(\mathbf{x})}, \mathbf{s} \in \mathcal{V}_{\sigma} \cap \mathcal{V}_{i n t}, \sigma \in \mathcal{F}_{\Gamma},
$$

constrained to satisfy $\alpha_{\sigma, \mathbf{s}} \geq 0$, and $\sum_{\mathbf{s} \in \mathcal{V}_{\sigma} \cap \mathcal{V}_{i n t}} \alpha_{\sigma, \mathbf{s}} \leq 1$. The convergence of the VAG scheme will be shown to hold whatever the choice of these partitions or volume fractions. As will be detailed in the numerical section, this flexibility is a crucial asset, compared with usual CVFE approaches, in order to improve the accuracy of the scheme for highly heterogeneous test cases.

Let $\rho_{T}$ denote the insphere diameter of a given tetrahedron $T, h_{T}$ its diameter, and $h_{\mathcal{T}}=$ $\max _{T \in \mathcal{T}} h_{T}$. We will assume in the convergence analysis that the family of tetrahedral submeshes $\mathcal{T}$ is shape regular and that the number of vertices of each cell $K$ is uniformly bounded. Hence let us set

$$
\theta_{\mathcal{T}}=\max _{T \in \mathcal{T}} \frac{h_{T}}{\rho_{T}} \quad \text { and } \quad \gamma_{\mathcal{M}}=\max _{K \in \mathcal{M}} \operatorname{Card}\left(\mathcal{V}_{K}\right) .
$$

We state without proof two results that can be readily adapted from [6] noticing that the shape regularity of $\mathcal{T}$ implies the shape regularity of the triangular submesh of $\Gamma$ defined by $\mathcal{T} \cap \Gamma$.

Lemma 2.1 There exist $C_{1}, C_{2}>0$ depending only on $\gamma_{\mathcal{M}}$ and $\theta_{\mathcal{T}}$ such that for all $u \in X_{\mathcal{D}}$

$$
\left\|\pi_{\mathcal{D}_{m}} u\right\|_{\mathrm{L}^{2}(\Omega)} \leq C_{1}\left\|\pi_{\mathcal{T}} u\right\|_{\mathrm{L}^{2}(\Omega)} \quad \text { and } \quad\left\|\pi_{\mathcal{D}_{f}} u\right\|_{\mathrm{L}^{2}(\Gamma)} \leq C_{2}\left\|\gamma \pi_{\mathcal{T}} u\right\|_{\mathrm{L}^{2}(\Gamma)} .
$$


Lemma 2.2 There exists $C_{3}>0$ depending only on $\gamma_{\mathcal{M}}$ and $\theta_{\mathcal{T}}$ such that, for all $u \in X_{\mathcal{D}}$,

$$
\left\|\pi_{\mathcal{D}_{m}} u-\pi_{\mathcal{T}} u\right\|_{L^{2}(\Omega)}+\left\|\pi_{\mathcal{D}_{f}} u-\gamma \pi_{\mathcal{T}} u\right\|_{L^{2}(\Gamma)} \leq C_{3} h_{\mathcal{T}}\left\|\pi_{\mathcal{T}} u\right\|_{V}
$$

For any smooth function $\varphi \in C^{\infty}(\bar{\Omega})$, let us introduce the finite element interpolation operator

$$
P_{\mathcal{T}} \varphi(\mathbf{x})=\sum_{K \in \mathcal{M}} \varphi\left(\mathbf{x}_{K}\right) \eta_{K}(\mathbf{x})+\sum_{\mathbf{s} \in \mathcal{V}} \varphi\left(\mathbf{x}_{\mathbf{s}}\right) \eta_{\mathbf{s}}(\mathbf{x})+\sum_{\sigma \in \mathcal{F}_{\Gamma}} \varphi\left(\mathbf{x}_{\sigma}\right) \eta_{\sigma}(\mathbf{x})
$$

We have the following classical finite element approximation result:

Proposition 2.1 For all $\varphi \in C^{\infty}(\bar{\Omega})$, then there exists $C_{4}>0$ depending only on $\varphi, \gamma_{\mathcal{M}}$, and $\theta_{\mathcal{T}}$ such that

$$
\left\|\varphi-P_{\mathcal{T}} \varphi\right\|_{V} \leq C_{4} h_{\mathcal{T}}
$$

The VAG scheme has been introduced for the discretization of multiphase immiscible Darcy flows in [14] and in [15] for compositional models. Its convergence has been proved for two-phase flows using a global pressure formulation in [6]. In [17] it has been adapted to take into account discontinuous capillary pressures using a phase pressures formulation and the convergence proof is done in the general framework of gradient schemes. We extend here this approach to the case of hybrid dimensional two-phase Darcy flows.

For $N \in \mathbb{N}^{*}$, let us consider the time discretization $t^{0}=0<t^{1}<\cdots<t^{n-1}<t^{n} \cdots<t^{N}=T$ of the time interval $[0, T]$. We denote the time steps by $\Delta t^{n}=t^{n}-t^{n-1}$ for all $n=1, \cdots, N$ while $\Delta t$ stands for the whole sequence $\left(\Delta t^{n}\right)_{n=1, \ldots, N}$.

Let us denote by $u^{\alpha, n} \in X_{\mathcal{D}}^{0}, \alpha=1,2$ the discrete phase pressures, and by $p^{n}=u^{1, n}-u^{2, n}$ the discrete capillary pressure at time $t^{n}$ for all $n=1, \cdots, N$. Given an approximation $p^{0} \in X_{\mathcal{D}}$ of the initial capillary pressure $p_{\text {ini }}$, the VAG discretization of the two-phase Darcy flow model in phase pressures formulation (1) looks for $u^{\alpha}=\left(u^{\alpha, n} \in X_{\mathcal{D}}^{0}\right)_{n=1, \cdots, N}, \alpha=1,2$, such that for $\alpha=1,2$, and for all $v \in X_{\mathcal{D}}^{0}$ one has

$$
\left\{\begin{aligned}
\int_{\Omega} \phi_{m} \frac{S_{\mathcal{D}_{m}}^{\alpha, n}-S_{\mathcal{D}_{m}}^{\alpha, n-1}}{\Delta t^{n}} \pi_{\mathcal{D}_{m}} v d \mathbf{x} & +\int_{\Omega} k_{\mathcal{D}_{m}}^{\alpha, n} \Lambda_{m} \nabla_{\mathcal{D}_{m}} u^{\alpha, n} \cdot \nabla_{\mathcal{D}_{m}} v d \mathbf{x} \\
+\int_{\Gamma} \phi_{f} \frac{S_{\mathcal{D}_{f}}^{\alpha, n}-S_{\mathcal{D}_{f}}^{\alpha, n-1}}{\Delta t^{n}} \pi_{\mathcal{D}_{f}} v d \tau_{f}(\mathbf{x}) & +\int_{\Gamma} k_{\mathcal{D}_{f}}^{\alpha, n} \Lambda_{f} \nabla_{\mathcal{D}_{f}} u^{\alpha, n} \cdot \nabla_{\mathcal{D}_{f}} v d \tau_{f}(\mathbf{x}) \\
& =\frac{1}{\Delta t^{n}} \int_{t^{n-1}}^{t^{n}}\left(\int_{\Omega} h_{m}^{\alpha} \pi_{\mathcal{D}_{m}} v d \mathbf{x}+\int_{\Gamma} h_{f}^{\alpha} \pi_{\mathcal{D}_{f}} v d \tau_{f}(\mathbf{x})\right) d t
\end{aligned}\right.
$$

where the saturations and relative permeabilities are discretized using the piecewise constant reconstruction operators

$$
S_{\mathcal{D}_{m}}^{\alpha, n}(\mathbf{x})=S_{m}^{\alpha}\left(\mathbf{x}, \pi_{\mathcal{D}_{m}} p^{n}(\mathbf{x})\right), S_{\mathcal{D}_{f}}^{\alpha, n}(\mathbf{x})=S_{f}^{\alpha}\left(\mathbf{x}, \pi_{\mathcal{D}_{f}} p^{n}(\mathbf{x})\right),
$$

and

$$
k_{\mathcal{D}_{m}}^{\alpha, n}(\mathbf{x})=k_{m}^{\alpha}\left(\mathbf{x}, S_{\mathcal{D}_{m}}^{\alpha, n}(\mathbf{x})\right), k_{\mathcal{D}_{f}}^{\alpha, n}(\mathbf{x})=k_{f}^{\alpha}\left(\mathbf{x}, S_{\mathcal{D}_{f}}^{\alpha, n}(\mathbf{x})\right),
$$

in order to capture the discontinuities at different rocktype interfaces. 


\section{Convergence Analysis}

\subsection{A priori estimates}

Using the phase pressures as test functions in the discrete variational formulation (4), we deduce the following a priori estimate.

Lemma 3.1 Assuming that hypotheses (H1 - H5) hold, let $u^{\alpha}, \alpha=1,2$, be a solution to (4), then, there exists $C_{5}>0$ depending only on the data and on $\gamma_{\mathcal{M}}$ and $\theta_{\mathcal{T}}$ such that

$$
\sum_{\alpha=1,2} \sum_{n=1}^{N} \Delta t^{n}\left\|\pi_{\mathcal{T}} u^{\alpha, n}\right\|_{V}^{2} \leq C_{5}
$$

Let us introduce the following notations. For all $v \in X_{\mathcal{D}}$ we define the terms

$$
\begin{array}{cc}
A_{\mathcal{D}_{m}}^{\alpha, n}(v)=\int_{\Omega} \phi_{m} \frac{S_{\mathcal{D}_{m}}^{\alpha, n}-S_{\mathcal{D}_{m}}^{\alpha, n-1}}{\Delta t^{n}} \pi_{\mathcal{D}_{m}} v d \mathbf{x}, & A_{\mathcal{D}_{f}}^{\alpha, n}(v)=\int_{\Gamma} \phi_{f} \frac{S_{\mathcal{D}_{f}}^{\alpha, n}-S_{\mathcal{D}_{f}}^{\alpha, n-1}}{\Delta t^{n}} \pi_{\mathcal{D}_{f}} v d \tau_{f}(\mathbf{x}), \\
B_{\mathcal{D}_{m}}^{\alpha, n}(v)=\int_{\Omega} k_{\mathcal{D}_{m}}^{\alpha, n} \Lambda_{m} \nabla_{\mathcal{D}_{m}} u^{\alpha, n} \cdot \nabla_{\mathcal{D}_{m}} v d \mathbf{x}, & B_{\mathcal{D}_{f}}^{\alpha, n}(v)=\int_{\Gamma} k_{\mathcal{D}_{f}}^{\alpha, n} \Lambda_{f} \nabla_{\mathcal{D}_{f}} u^{\alpha, n} \cdot \nabla_{\mathcal{D}_{f}} v d \tau_{f}(\mathbf{x}), \\
C_{\mathcal{D}_{m}}^{\alpha, n}(v)=\frac{1}{\Delta t^{n}} \int_{t^{n-1}}^{t^{n}} \int_{\Omega} h_{m}^{\alpha} \pi_{\mathcal{D}_{m}} v d \mathbf{x} d t, & C_{\mathcal{D}_{f}}^{\alpha, n}(v)=\frac{1}{\Delta t^{n}} \int_{t^{n-1}}^{t^{n}} \int_{\Gamma} h_{f}^{\alpha} \pi_{\mathcal{D}_{f}} v d \tau_{f}(\mathbf{x}) d t .
\end{array}
$$

In order to prove Lemma 3.1, we first derive some estimates of the accumulation, gradient and right hand side terms of in the following propositions.

Firstly, the following estimate of the accumulation terms is a straightforward adaptation from Lemma 3.1 of [17].

Proposition 3.1 Let $u^{\alpha}$, $\alpha=1,2$, be a solution of (4), then

$$
\sum_{n=1}^{N} \sum_{\alpha=1,2} \sum_{j=m, f} \Delta t^{n} A_{\mathcal{D}_{j}}^{\alpha, n}\left(u^{\alpha, n}\right) \geq-\frac{\phi_{\max } L_{S}}{2}\left(\left\|\pi_{\mathcal{D}_{m}} p^{0}\right\|_{L^{2}(\Omega)}^{2}+d_{\max }\left\|\pi_{\mathcal{D}_{f}} p^{0}\right\|_{L^{2}(\Gamma)}^{2}\right) .
$$

Secondly, thanks to the assumptions (H2) and (H4) the following estimate readily holds for the gradient terms.

Proposition 3.2 Let $u^{\alpha}, \alpha=1,2$, be a solution of (4), then

$$
\sum_{n=1}^{N} \sum_{j=m, f} \Delta t^{n} B_{\mathcal{D}_{j}}^{\alpha, n}\left(u^{\alpha, n}\right) \geq k_{\min } \lambda_{\min } \sum_{n=1}^{N} \Delta t^{n}\left(\left\|\nabla_{\mathcal{D}_{m}} u^{\alpha, n}\right\|_{\left.L^{2} \Omega\right)^{d}}^{2}+d_{\min }\left\|\nabla_{\mathcal{D}_{f}} u^{\alpha, n}\right\|_{L^{2}(\Gamma)^{d-1}}^{2}\right),
$$

for all $\alpha=1,2$.

Thirdly, we have the following straightforward estimate for the right hand side.

Proposition 3.3 Let $u^{\alpha}, \alpha=1,2$, be a solution of (4), then there exists $C>0$ depending only on $d_{\max }, C_{P}, \gamma_{\mathcal{M}}$ and $\theta_{\mathcal{T}}$ such that

$$
\sum_{n=1}^{N} \sum_{j=m, f} \Delta t^{n} C_{\mathcal{D}_{j}}^{\alpha, n}\left(u^{\alpha, n}\right) \leq C\left(\left\|h_{m}^{\alpha}\right\|_{L^{2}(\Omega \times(0, T))}^{2}+d_{\max }\left\|h_{f}^{\alpha}\right\|_{L^{2}(\Gamma \times(0, T))}^{2}\right)^{1 / 2}\left(\sum_{n=1}^{N} \Delta t^{n}\left\|\pi_{\mathcal{T}} u^{\alpha, n}\right\|_{V}^{2}\right)^{1 / 2}
$$

for all $\alpha=1,2$. 
Proof of Lemma 3.1: To complete the proof of Lemma 3.1, it follows from (4) that

$$
\sum_{n=1}^{N} \sum_{\alpha=1,2} \sum_{j=m, f} \Delta t^{n}\left(A_{\mathcal{D}_{j}}^{\alpha, n}\left(u^{\alpha, n}\right)+B_{\mathcal{D}_{j}}^{\alpha, n}\left(u^{\alpha, n}\right)-C_{\mathcal{D}_{j}}^{\alpha, n}\left(u^{\alpha, n}\right)\right)=0,
$$

so that in view of Propositions 3.1, 3.2 and 3.3 there exists $C>0$ depending only on the data and on $\gamma_{\mathcal{M}}$ and $\theta_{\mathcal{T}}$ such that

$$
\sum_{\alpha=1,2} \sum_{n=1}^{N} \Delta t^{n}\left\|\pi_{\mathcal{T}} u^{\alpha, n}\right\|_{V}^{2} \leq C\left(1+\left(\sum_{\alpha=1,2} \sum_{n=1}^{N} \Delta t^{n}\left\|\pi_{\mathcal{T}} u^{\alpha, n}\right\|_{V}^{2}\right)^{1 / 2}\right) .
$$

The estimate (5) is then obtained using Young's inequality.

Lemma 3.2 Assuming that hypotheses $(\mathrm{H} 1-\mathrm{H} 5)$ hold, there exists at least one solution to the problem (4).

Proof: Let us consider the functions $S_{m}^{1, \theta}=\theta S_{m}^{1}+(1-\theta)$ and $S_{f}^{1, \theta}=\theta S_{f}^{1}+(1-\theta)$ instead of $S_{m}^{1}$ and $S_{f}^{1}$ as well as the functions $S_{m}^{2, \theta}=1-S_{m}^{1, \theta}$ and $S_{f}^{2, \theta}=1-S_{f}^{1, \theta}$ instead of $S_{m}^{2}$ and $S_{f}^{2}$. The problem corresponding to $\theta=0$ reads : For each $\alpha=1,2$ and for each $n=1, \ldots, N$ find $u^{\alpha, n} \in X_{\mathcal{D}}^{0}$ satisfying

$$
\left\{\begin{array}{c}
\int_{\Omega} k_{m}^{1}(\mathbf{x}, 1) \Lambda_{m} \nabla_{\mathcal{D}_{m}} u^{1, n} \cdot \nabla_{\mathcal{D}_{m}} v d \mathbf{x}+\int_{\Gamma} k_{f}^{1}(\mathbf{x}, 1) \Lambda_{f} \nabla_{\mathcal{D}_{f}} u^{1, n} \cdot \nabla_{\mathcal{D}_{f}} v d \tau_{f}(\mathbf{x}) \\
=\frac{1}{\Delta t^{n}} \int_{t^{n-1}}^{t^{n}}\left(\int_{\Omega} h_{m}^{1} v d \mathbf{x}+\int_{\Gamma} h_{f}^{1} \pi_{\mathcal{D}_{f}} v d \tau_{f}(\mathbf{x})\right) d t \quad \forall v \in X_{\mathcal{D}}^{0} \\
\int_{\Omega} k_{m}^{2}(\mathbf{x}, 0) \Lambda_{m} \nabla_{\mathcal{D}_{m}} u^{2, n} \cdot \nabla_{\mathcal{D}_{m}} v d \mathbf{x}+\int_{\Gamma} k_{f}^{2}(\mathbf{x}, 0) \Lambda_{f} \nabla_{\mathcal{D}_{f}} u^{2, n} \cdot \nabla_{\mathcal{D}_{f}} v d \tau_{f}(\mathbf{x}) \\
=\frac{1}{\Delta t^{n}} \int_{t^{n-1}}^{t^{n}}\left(\int_{\Omega} h_{m}^{2} v d \mathbf{x}+\int_{\Gamma} h_{f}^{2} \pi_{\mathcal{D}_{f}} v d \tau_{f}(\mathbf{x})\right) d t \quad \forall v \in X_{\mathcal{D}}^{0} .
\end{array}\right.
$$

In view of Lemma 2.1 and the assumptions on the data one deduces from Lax-Milgram theorem the existence and uniqueness of the solution to (9). We remark that the estimate (5) holds for all $\theta \in[0,1]$. Therefore the existence of a solution for all $\theta \in[0,1]$ can be deduced from a classical topological degree argument.

\subsection{Estimates on the time and space translates}

Proposition 3.4 Let $T>0, N \in \mathbb{N}$ and $\left(t^{n}\right)_{n=0, \ldots, N} \in \mathbb{R}$ such that $0=t^{0}<t_{1}<\ldots<t^{N}=T$. Let $v$ be a piecewise constant mapping from $[0, T]$ to some space $X$ (endowed with a semi-norm $\|\cdot\|_{X}$ ) such that $v(0)=v^{0}$ and $v(t)=v^{n}$ for all $t \in\left(t^{n-1}, t^{n}\right]$. Then,

$$
\int_{0}^{T-\tau}\|v(t+\tau)-v(t)\|_{X} \leq \tau \sum_{n=1}^{N}\left\|v^{n}-v^{n-1}\right\|_{X} \quad \forall \tau \in[0, T] .
$$

Proof: For all $t \in[0, T]$ we set

$$
n_{\Delta t}(t)= \begin{cases}0 & \text { if } \quad t=0 \\ n & \text { if } \quad t \in\left(t_{n-1}, t_{n}\right]\end{cases}
$$


so that

$$
\int_{0}^{T-\tau}\|v(t+\tau)-v(t)\|_{X} d t \leq \int_{0}^{T-\tau} \sum_{k=n_{\Delta t}(t)+1}^{n_{\Delta t}(t+\tau)}\left\|v^{k}-v^{k-1}\right\|_{X} d t .
$$

We conclude the proof by applying Lemma 6.1 of [2].

Let us set $X_{\mathcal{D}, \Delta t}=\left(X_{\mathcal{D}}\right)^{N}$, and for all $v=\left(v^{n}\right)_{n=1, \cdots, N} \in X_{\mathcal{D}, \Delta t}$ let us define

$$
\begin{aligned}
& \pi_{\mathcal{D}_{m}, \Delta t} v(\mathbf{x}, t)=\pi_{\mathcal{D}_{m}} v^{n}(\mathbf{x}) \text { for all }(\mathbf{x}, t) \in \Omega \times\left(t^{n-1}, t^{n}\right], \\
& \pi_{\mathcal{D}_{f}, \Delta t} v(\mathbf{x}, t)=\pi_{\mathcal{D}_{f}} v^{n}(\mathbf{x}) \text { for all }(\mathbf{x}, t) \in \Gamma \times\left(t^{n-1}, t^{n}\right] \\
& \pi_{\mathcal{T}, \Delta t} v(\mathbf{x}, t)=\pi_{\mathcal{T}} v^{n}(\mathbf{x}) \text { for all }(\mathbf{x}, t) \in \Omega \times\left(t^{n-1}, t^{n}\right]
\end{aligned}
$$

We also define the functions $S_{\mathcal{D}_{m}, \Delta t}^{\alpha}(\mathbf{x}, t)=S^{\alpha}\left(\mathbf{x}, \pi_{\mathcal{D}_{m}, \Delta t} p(\mathbf{x}, t)\right)$ and $S_{\mathcal{D}_{f}, \Delta t}^{\alpha}(\mathbf{x}, t)=S^{\alpha}\left(\mathbf{x}, \pi_{\mathcal{D}_{f}, \Delta t} p(\mathbf{x}, t)\right)$.

Lemma 3.3 Assuming that hypotheses (H1 - H5) hold, let $u^{\alpha}, \alpha=1,2$, be a solution to (4), then there exists $C>0$ only depending on the data, on $\gamma_{\mathcal{M}}$ and $\theta_{\mathcal{T}}$ such that for all $\tau>0$ one has

$$
\sum_{\alpha=1,2}\left\|S_{\mathcal{D}_{m}, \Delta t}^{\alpha}(\cdot, \cdot+\tau)-S_{\mathcal{D}_{m}, \Delta t}^{\alpha}\right\|_{L^{2}(\Omega \times(0, T))}^{2}+\left\|S_{\mathcal{D}_{f}, \Delta t}^{\alpha}(\cdot, \cdot+\tau)-S_{\mathcal{D}_{f}, \Delta t}^{\alpha}\right\|_{L^{2}(\Gamma \times(0, T))}^{2} \leq C \sqrt{\tau},
$$

where we have set $S_{\mathcal{D}_{m}, \Delta t}^{\alpha}(\mathbf{x}, t)=S_{\mathcal{D}_{f}, \Delta t}^{\alpha}(\mathbf{x}, t)=0$ for all $t>T$.

Proof: Let us denote by $U$ the space $L^{2}(\Omega) \times L^{2}(\Gamma)$ equipped with the scalar product $\langle u, v\rangle_{U}=$ $\int_{\Omega} \phi_{m} u_{m} v_{m} d \mathbf{x}+\int_{\Gamma} \phi_{f} u_{f} v_{f} d \tau_{f}(\mathbf{x})$. For all $u=\left(u_{m}, u_{f}\right) \in U$ we also define the dual semi-norm $\|u\|_{-1, \mathcal{D}}$ by

$$
\|u\|_{-1, \mathcal{D}}=\sup _{v \in X_{\mathcal{D}}^{0}, v \neq 0} \frac{\left\langle u,\left(\pi_{\mathcal{D}_{m}} v, \pi_{\mathcal{D}_{f}} v\right)\right\rangle_{U}}{\left\|\pi_{\mathcal{T}} v\right\|_{V}} .
$$

Setting $S_{\mathcal{D}}^{\alpha, n}=\left(S_{\mathcal{D}_{m}}^{\alpha, n}, S_{\mathcal{D}_{f}}^{\alpha, n}\right) \in U$, and using the assumptions $\left(\mathrm{H}_{1}\right),\left(\mathrm{H}_{2}\right),\left(\mathrm{H}_{4}\right),\left(\mathrm{H}_{5}\right)$ on the data, we deduce from (4) that there exists $C>0$ depending only on the data and on $\gamma_{\mathcal{M}}$ and $\theta_{\mathcal{T}}$ such that

$\left|\frac{1}{\Delta t^{n}}\left\langle S_{\mathcal{D}}^{\alpha, n}-S_{\mathcal{D}}^{\alpha, n-1},\left(\pi_{\mathcal{D}_{m}} v, \pi_{\mathcal{D}_{f}} v\right)\right\rangle_{U}\right| \leq C\left(\left\|\pi_{\mathcal{T}} u^{\alpha, n}\right\|_{V}+\frac{1}{\Delta t^{n}} \int_{t^{n-1}}^{t^{n}}\left(\left\|h_{m}(., t)\right\|_{L^{2}(\Omega)}+\left\|h_{f}(., t)\right\|_{L^{2}(\Gamma)}\right) d t\right)\left\|\pi_{\mathcal{T}} v\right\|_{V}$

for all $v \in X_{\mathcal{D}}^{0}$. Therefore, one obtains the estimate

$$
\frac{1}{\Delta t^{n}}\left\|S_{\mathcal{D}}^{\alpha, n}-S_{\mathcal{D}}^{\alpha, n-1}\right\|_{-1, \mathcal{D}} \leq C\left(\left\|\pi_{\mathcal{T}} u^{\alpha, n}\right\|_{V}+\frac{1}{\Delta t^{n}} \int_{t^{n-1}}^{t^{n}}\left(\left\|h_{m}(., t)\right\|_{L^{2}(\Omega)}+\left\|h_{f}(., t)\right\|_{L^{2}(\Gamma)}\right) d t\right) .
$$

Multiplying by $\Delta t^{n}$, summing over $n=1, \ldots, N$, and using Lemma 3.1, there exists $C>0$ depending only on the data and on $\gamma_{\mathcal{M}}$ and $\theta_{\mathcal{T}}$ such that

$$
\sum_{n=1}^{N}\left\|S_{\mathcal{D}}^{\alpha, n}-S_{\mathcal{D}}^{\alpha, n-1}\right\|_{-1, \mathcal{D}} \leq C .
$$

Next setting $p=u^{1}-u^{2}$ and $S_{\mathcal{D}, \Delta t}^{\alpha, n}=\left(S_{\mathcal{D}_{m}, \Delta t}^{\alpha}, S_{\mathcal{D}_{f}, \Delta t}^{\alpha}\right) \in L^{2}(U \times(0, T))$ we have

$$
\begin{aligned}
& \int_{0}^{T}\left\|S_{\mathcal{D}, \Delta t}^{\alpha}(t+\tau)-S_{\mathcal{D}, \Delta t}^{\alpha}(t)\right\|_{U} d t \\
& \leq \sqrt{L_{S}} \int_{0}^{T}\left\|S_{\mathcal{D}, \Delta t}^{\alpha}(t+\tau)-S_{\mathcal{D}, \Delta t}^{\alpha}(t)\right\|_{-1, \mathcal{D}}^{1 / 2}\left\|\pi_{\mathcal{T}, \Delta t} p(t+\tau)-\pi_{\mathcal{T}, \Delta t} p(t)\right\|_{V}^{1 / 2} d t \\
& \leq \frac{\sqrt{L_{S}}}{2 \sqrt{\tau}} \int_{0}^{T}\left\|S_{\mathcal{D}, \Delta t}^{\alpha}(t+\tau)-S_{\mathcal{D}, \Delta t}^{\alpha}(t)\right\|_{-1, \mathcal{D}} d t+\frac{\sqrt{L_{S}} \sqrt{\tau}}{2} \int_{0}^{T}\left\|\pi_{\mathcal{T}, \Delta t} p(t+\tau)-\pi_{\mathcal{T}, \Delta t} p(t)\right\|_{V} d t .
\end{aligned}
$$


In view of Proposition 3.4, the estimates (10), Lemma 3.1, and the assumption $\left(\mathrm{H}_{3}\right)$, there exists $C>0$ depending only on the data and on $\gamma_{\mathcal{M}}$ and $\theta_{\mathcal{T}}$ such that

$$
\frac{\sqrt{L_{S}}}{2 \sqrt{\tau}} \int_{0}^{T}\left\|S_{\mathcal{D}, \Delta t}^{\alpha}(t+\tau)-S_{\mathcal{D}, \Delta t}^{\alpha}(t)\right\|_{-1, \mathcal{D}} d t \leq C \sqrt{\tau}
$$

and

$$
\int_{0}^{T}\left\|\pi_{\mathcal{T}, \Delta t} p(t+\tau)-\pi_{\mathcal{T}, \Delta t} p(t)\right\|_{V} d t \leq C
$$

which implies that

$$
\int_{0}^{T}\left\|S_{\mathcal{D}, \Delta t}^{\alpha}(t+\tau)-S_{\mathcal{D}, \Delta t}^{\alpha}(t)\right\|_{U} d t \leq C \sqrt{\tau}
$$

with $C>0$ depending only on the data and on $\gamma_{\mathcal{M}}$ and $\theta_{\mathcal{T}}$. One concludes the proof using $0 \leq$ $S_{m}^{\alpha}, S_{f}^{\alpha} \leq 1$.

Lemma 3.4 It is assumed that hypotheses $(\mathrm{H} 1-\mathrm{H} 5)$ hold. Let $\left(\mathcal{D}^{(m)}, \Delta t^{(m)}\right)_{m \in \mathbb{N}}$ be a sequence of space-time discretizations such that there exist two positive constants $\theta$ and $\gamma$ satisfying $\theta_{\mathcal{T}^{(m)}} \leq \theta$, $\gamma_{\mathcal{M}^{(m)}} \leq \gamma$ for all $m \in \mathbb{N}$ and such that $h_{\mathcal{T}^{(m)}} \rightarrow 0$ as $m \rightarrow \infty$. Let $u^{\alpha,(m)}, S_{\mathcal{D}_{m}^{(m)}, \Delta t}^{\alpha(m)}$ and $S_{\mathcal{D}_{f}^{(m)}, \Delta t^{(m)}}^{\alpha}$, $\alpha=1,2$, be such that (4) holds for all $m \in \mathbb{N}$.

1. Let $\xi \in \mathbb{R}^{d}$ and

$$
T_{m}^{(m)}(\xi)=\sum_{\alpha=1,2}\left\|S_{\mathcal{D}_{m}^{(m)}, \Delta t^{(m)}}^{\alpha}(\cdot+\xi, \cdot)-S_{\mathcal{D}_{m}^{(m)}, \Delta t^{(m)}}^{\alpha}\right\|_{L^{2}\left(\mathbb{R}^{d} \times(0, T)\right)},
$$

where $S_{\mathcal{D}_{m}^{(m)}, \Delta t^{(m)}}^{\alpha}$ is extended by 0 on $\left(\mathbb{R}^{d} \backslash \bar{\Omega}\right) \times(0, T)$. Then, one has $\lim _{|\xi| \rightarrow 0} \sup _{m \in \mathbb{N}} T_{m}^{(m)}(\xi)=$ 0 .

2. For all $i \in I$ let $\tau\left(\mathcal{P}_{i}\right)$ denote the vector subspace tangent to $\mathcal{P}_{i}$, let $\xi \in \tau\left(\mathcal{P}_{i}\right)$, and let us set

$$
T_{f}^{(m)}(\xi)=\sum_{\alpha=1,2}\left\|S_{\mathcal{D}_{f, i}^{(m)}, \Delta t^{(m)}}^{\alpha}(\cdot+\xi, \cdot)-S_{\mathcal{D}_{f, i}^{(m)}, \Delta t}^{\alpha}\right\|_{L^{2}\left(\mathcal{P}_{i} \times(0, T)\right)},
$$

where $S_{\mathcal{D}_{f, i}^{(m)}, \Delta t^{(m)}}^{\alpha}$ denotes the restriction of $S_{\mathcal{D}_{f}^{(m)}, \Delta t^{(m)}}^{\alpha}$ to $\Gamma_{i}$, extended by 0 on $\left(\mathcal{P}_{i} \backslash \bar{\Gamma}_{i}\right) \times(0, T)$. Then one has $\lim _{|\xi| \rightarrow 0} \sup _{m \in \mathbb{N}} T_{f}^{(m)}(\xi)=0$

Proof: For each $i \in I$ and for all $\xi \in \tau\left(\mathcal{P}_{i}\right)$ let us define the set $\Gamma_{i}^{\xi}=\left\{\mathbf{x} \in \Gamma_{i}, \mathbf{x}+\xi \in \Gamma_{i}\right\}$. Since $0 \leq S_{\mathcal{D}_{f, i}}^{\alpha} \leq 1$ there exists a positive $C$ depending only on the geometry of $\Gamma_{i}$ and on $T$ such that $\left\|S_{\mathcal{D}_{f, i}^{(m)}, \Delta t^{(m)}}^{\alpha}(\cdot+\xi, \cdot)-S_{\mathcal{D}_{f, i}^{(m)}, \Delta t^{(m)}}^{\alpha}\right\|_{L^{2}\left(\Gamma_{i} \times(0, T)\right)}^{2} \leq C|\xi|+\left\|S_{\mathcal{D}_{f, i}^{(m)}, \Delta t^{(m)}}^{\alpha}(\cdot+\xi, \cdot)-S_{\mathcal{D}_{f, i}^{(m)}, \Delta t^{(m)}}^{\alpha}\right\|_{L^{2}\left(\Gamma_{i}^{\xi} \times(0, T)\right)}^{2}$.

Denoting by $\pi_{\mathcal{D}_{f, i}^{(m)}, \Delta t^{(m)}} p^{(m)}$ the restriction of $\pi_{\mathcal{D}_{f}^{(m)}, \Delta t^{(m)}} p^{(m)}$ to $\Gamma_{i}$, one deduces from Lemma 2.2 and Lemma 3.1 that

$$
\begin{aligned}
\| S_{\mathcal{D}_{f, i}^{(m)}, \Delta t^{(m)}}^{\alpha}(\cdot+\xi, \cdot) & -S_{\mathcal{D}_{f, i}^{(m)}, \Delta t^{(m)}}^{\alpha} \|_{L^{2}\left(\Gamma_{i}^{\xi} \times(0, T)\right)} \\
& \leq L_{S}\left\|\pi_{\mathcal{D}_{f, i}^{(m)}, \Delta t^{(m)}} p^{(m)}(\cdot+\xi, \cdot)-\pi_{\mathcal{D}_{f, i}^{(m)}, \Delta t^{(m)}} p^{(m)}\right\|_{L^{2}\left(\Gamma_{i}^{\xi} \times(0, T)\right)} \\
& \leq L_{S}\left\|\gamma_{i} \pi_{\mathcal{T}^{(m)}, \Delta t^{(m)}} p^{(m)}(\cdot+\xi, \cdot)-\gamma_{i} \pi_{\mathcal{T}^{(m)}, \Delta t^{(m)}} p^{(m)}\right\|_{L^{2}\left(\Gamma_{i}^{\xi} \times(0, T)\right)}+2 L_{S} C_{3} \sqrt{C_{5}} h_{\mathcal{T}^{(m)}} .
\end{aligned}
$$

Therefore, using Lemma 5.2 in the Appendix and Lemma B.2 of [16], we deduce that

$$
\lim _{\xi \rightarrow 0} \sup _{m \in \mathbb{N}}\left\|S_{\mathcal{D}_{f, i}^{(m)}, \Delta t^{(m)}}^{\alpha}(\cdot+\xi, \cdot)-S_{\mathcal{D}_{f, i}^{(m)}, \Delta t^{(m)}}^{\alpha}\right\|_{L^{2}\left(\mathcal{P}_{i} \times(0, T)\right)}=0 \quad \forall i \in I .
$$

One proves the first statement of the lemma using similar arguments. 


\subsection{Convergence}

In view of Lemma 3.3 and Lemma 3.4, the Kolmogorov-Fréchet theorem allows to establish the following relative compactness result for the saturation.

Lemma 3.5 It is assumed that hypotheses $(\mathrm{H} 1-\mathrm{H} 5)$ hold. Let $\left(\mathcal{D}^{(m)}, \Delta t^{(m)}\right)_{m \in \mathbb{N}}$ be a sequence of space-time discretizations such that there exist two positive constants $\theta$ and $\gamma$ satisfying $\theta_{\mathcal{T}^{(m)}} \leq \theta$, $\gamma_{\mathcal{M}^{(m)}} \leq \gamma$ for all $m \in \mathbb{N}$ and such that $h_{\mathcal{T}^{(m)}}, \max \Delta t^{(m)} \rightarrow 0$ as $m \rightarrow \infty$. Let $u^{\alpha,(m)}, S_{\mathcal{D}_{m}^{(m)}, \Delta t^{(m)}}^{\alpha}$ and $S_{\mathcal{D}_{f}^{(m)}, \Delta t^{(m)}}^{\alpha}, \alpha=1,2$, be s.t (4) holds for all $m \in \mathbb{N}$. Then, for each $\alpha=1,2$ one has the following result.

1. The sequence $\left(S_{\mathcal{D}_{m}^{(m)} \Delta t^{(m)}}^{\alpha}\right)_{m \in \mathbb{N}}$ is relatively compact in $L^{2}(\Omega \times(0, T))$.

2. For each $i \in I$ the sequence $\left(S_{\mathcal{D}_{f, i}^{(m)}, \Delta t^{(m)}}^{\alpha}\right)_{m \in \mathbb{N}}$ is relatively compact in $L^{2}\left(\Gamma_{i} \times(0, T)\right)$.

The limit of the saturation can be identified thanks to the following result.

Lemma 3.6 It is assumed that hypotheses $(\mathrm{H} 1-\mathrm{H} 5)$ hold. Let $\left(\mathcal{D}^{(m)}, \Delta t^{(m)}\right)_{m \in \mathbb{N}}$ be a sequence of space-time discretizations such that there exist two positive constants $\theta$ and $\gamma$ satisfying $\theta_{\mathcal{T}^{(m)}} \leq \theta$, $\gamma_{\mathcal{M}^{(m)}} \leq \gamma$ for all $m \in \mathbb{N}$ and such that $h_{\mathcal{T}^{(m)}}, \max \Delta t^{(m)} \rightarrow 0$ as $m \rightarrow \infty$. Let $u^{\alpha,(m)}, S_{\mathcal{D}_{m}^{(m)}, \Delta t^{(m)}}^{\alpha}$ and $S_{\mathcal{D}_{f}^{(m)}, \Delta t(m)}^{\alpha}, \alpha=1,2$, be s.t (4) holds for all $m \in \mathbb{N}$. Then, there exists a function pair $\left(\bar{u}^{\alpha}\right)_{\alpha=1,2} \in$ $\left(L^{2}\left(0, T ; V^{0}\right)\right)^{2}$ such that up to a subsequence

$$
\pi_{\mathcal{T}^{(m)}, \Delta t^{(m)}} u^{\alpha,(m)} \rightarrow \bar{u}^{\alpha} \text { in } L^{2}(\Omega \times(0, T)) \text { and } \gamma \pi_{\mathcal{T}^{(m)}, \Delta t^{(m)}} u^{\alpha,(m)} \rightarrow \gamma \bar{u}^{\alpha} \text { in } L^{2}(\Gamma \times(0, T))
$$

as $m \rightarrow \infty$; moreover setting $\bar{p}=\bar{u}^{1}-\bar{u}^{2}$, one has

$$
S_{\mathcal{D}_{m}^{(m)}, \Delta t^{(m)}}^{\alpha} \rightarrow S_{m}^{\alpha}(., \bar{p}) \text { in } L^{2}(\Omega \times(0, T)) \text { and } S_{\mathcal{D}_{f}^{(m)}, \Delta t^{(m)}}^{\alpha} \rightarrow S_{f}^{\alpha}(., \gamma \bar{p}) \text { in } L^{2}(\Gamma \times(0, T))
$$

as $m \rightarrow \infty$.

Proof: The existence of the weak limit $\left(\bar{u}^{\alpha}\right)_{\alpha=1,2}$ follows from Lemma 3.1 and Lemma 5.1. In order to prove the second statement we remark that it follows from Lemma 2.2 that $\pi_{\mathcal{D}_{m}^{(m)}, \Delta t^{(m)}} u^{\alpha}$ and $\pi_{\mathcal{D}_{f}^{(m)}, \Delta t^{(m)}} u^{\alpha}$ also converge weakly to $\bar{u}^{\alpha}$ in $L^{2}(\Omega \times(0, T))$ and to $\gamma \bar{u}^{\alpha}$ in $\left.L^{2}(\Gamma \times(0, T))\right)$ respectively. From Lemma 3.5, there exist four functions $\bar{s}_{m}^{\alpha} \in L^{2}(\Omega \times(0, T))$ and $\bar{s}_{f}^{\alpha} \in L^{2}(\Gamma \times(0, T)), \alpha=1,2$, with $\bar{s}_{m}^{1}+\bar{s}_{m}^{2}=1, \bar{s}_{f}^{1}+\bar{s}_{f}^{2}=1$, such that, up to a subsequence, $S_{\mathcal{D}_{m}^{(m)} \Delta t}^{\alpha}$ converges strongly to $\bar{s}_{m}^{\alpha}$ in $L^{2}(\Omega \times(0, T))$, and $S_{\mathcal{D}_{f}^{(m)} \Delta t^{(m)}}^{\alpha}$ converges strongly to $\bar{s}_{f}^{\alpha}$ in $L^{2}(\Gamma \times(0, T))$. Then, one can conclude the proof using the Minty trick stated in Lemma 3.6 of [17] to show that $\bar{s}_{m}^{\alpha}=S_{m}^{\alpha}\left(., \bar{u}^{1}-\bar{u}^{2}\right)$ and $\bar{s}_{f}^{\alpha}=S_{f}^{\alpha}\left(., \gamma\left(\bar{u}^{1}-\bar{u}^{2}\right)\right)$.

Theorem 3.1 It is assumed that hypotheses $(\mathrm{H} 1-\mathrm{H} 5)$ hold. Let $\left(\mathcal{D}^{(m)}, \Delta t^{(m)}\right)_{m \in \mathbb{N}}$ be a sequence of space-time discretizations such that there exist two positive constants $\theta$ and $\gamma$ satisfying $\theta_{\mathcal{T}^{(m)}} \leq \theta$, $\gamma_{\mathcal{M}^{(m)}} \leq \gamma$ for all $m \in \mathbb{N}$ and such that $h_{\mathcal{T}^{(m)}}, \max _{n} \Delta t^{(m), n} \rightarrow 0$ as $m \rightarrow \infty$. Let $u^{\alpha,(m)}, S_{\mathcal{D}_{m}^{(m)}, \Delta t^{(m)}}^{\alpha}$ and $S_{\mathcal{D}_{f}^{(m)}, \Delta t^{(m)}}^{\alpha}, \alpha=1,2$, be s.t (4) holds for all $m \in \mathbb{N}$. It is also assumed that $\pi_{\mathcal{D}_{m}^{(m)}} p^{0,(m)}$ converges strongly to $p^{\text {ini }}$ in $L^{2}(\Omega)$, and that $\pi_{\mathcal{D}_{f}^{(m)}} p^{0,(m)}$ converges strongly to $\gamma p^{\mathrm{ini}}$ in $L^{2}(\Gamma)$. Then there exists a weak solution $\left(\bar{u}^{1}, \bar{u}^{2}\right)$ to the problem (1) such that for each phase $\alpha=1,2$

$$
\pi_{\mathcal{T}^{(m)}, \Delta t^{(m)}} u^{\alpha,(m)} \rightarrow \bar{u}^{\alpha} \text { in } L^{2}(\Omega \times(0, T)) \text { and } \gamma \pi_{\mathcal{T}^{(m)}, \Delta t^{(m)}} u^{\alpha,(m)} \rightarrow \gamma \bar{u}^{\alpha} \text { in } L^{2}(\Gamma \times(0, T))
$$

up to a subsequence. 
Proof: For all $\alpha=1,2$ we denote by $\bar{u}^{\alpha}$ a weak limit of $\pi_{\mathcal{T}^{(m)}, \Delta t^{(m)}} u^{\alpha,(m)}$, whose existence is stated by Lemma 3.6. We show below that $\left(\bar{u}^{1}, \bar{u}^{2}\right)$ satisfies the variational formulation (1). In order to simplify the notation we drop the index $(m)$.

Let $\bar{\psi}$ be an arbitrary function from $C_{c}^{\infty}(\Omega \times[0, T))$ and $\psi(t)$ the projection of $\bar{\psi}(., t)$ to $X_{\mathcal{D}}^{0}$ defined by $\psi_{\nu}(t)=\psi\left(\mathbf{x}_{\nu}, t\right)$ for all $\nu \in \mathcal{M} \cup \mathcal{V} \cup \mathcal{F}_{\Gamma}$. Taking $v=\psi\left(t^{n-1}\right)$ in (4) for all $n=1, \ldots, N$ and summing over $n=1, \ldots, N$ we obtain that

$$
\sum_{n=1}^{N} \sum_{j=m, f} \Delta t^{n}\left(A_{\mathcal{D}_{j}}^{\alpha, n}\left(\psi\left(t^{n-1}\right)\right)+B_{\mathcal{D}_{j}}^{\alpha, n}\left(\psi\left(t^{n-1}\right)\right)\right)=\sum_{n=1}^{N} \sum_{j=m, f} \Delta t^{n} C_{\mathcal{D}_{j}}^{\alpha, n}\left(\psi\left(t^{n-1}\right)\right) .
$$

for each phase $\alpha=1,2$.

Accumulation terms. Let us consider the term $\sum_{n=1}^{N} \Delta t^{n} A_{\mathcal{D}_{m}}^{\alpha, n}\left(\psi\left(t^{n-1}\right)\right)$. Applying the chain rule we obtain that

$$
\sum_{n=1}^{N} \Delta t^{n} A_{\mathcal{D}_{m}}^{\alpha, n}\left(\psi\left(t^{n-1}\right)\right)=-\sum_{n=1}^{N} \int_{t^{n-1}}^{t^{n}} \int_{\Omega} \phi_{m} S_{\mathcal{D}_{m}}^{\alpha, n} \partial_{t} \pi_{\mathcal{D}_{m}} \psi(t) d \mathbf{x} d t-\int_{\Omega} \phi_{m} S_{\mathcal{D}_{m}}^{\alpha, 0} \pi_{\mathcal{D}_{m}} \psi\left(t^{0}\right) d \mathbf{x} .
$$

Thanks to the strong convergence of the function $S_{\mathcal{D}_{m}, \Delta t}^{\alpha}$ given by Lemma 3.6, to the regularity of $\bar{\psi}$, and to the convergence of $\pi_{\mathcal{D}_{m}} p^{0}$, one deduces that

$$
\sum_{n=1}^{N} \Delta t^{n} A_{\mathcal{D}_{m}}^{\alpha, n}\left(\psi\left(t^{n-1}\right)\right) \rightarrow-\int_{0}^{T} \int_{\Omega} \phi_{m} S_{m}^{\alpha}(., \bar{p}) \partial_{t} \bar{\psi} d \mathbf{x} d t-\int_{\Omega} \phi_{m} S_{m}^{\alpha}\left(., p_{i n i}\right) \bar{\psi}(\mathbf{x}, 0) d \mathbf{x} d t .
$$

Similarly we obtain that

$$
\sum_{n=1}^{N} \Delta t^{n} A_{\mathcal{D}_{f}}^{\alpha, n}\left(\psi\left(t^{n-1}\right)\right) \rightarrow-\int_{0}^{T} \int_{\Gamma} \phi_{f} S_{f}^{\alpha}(., \gamma \bar{p}) \partial_{t} \gamma \bar{\psi} d \tau_{f}(\mathbf{x}) d t-\int_{\Gamma} \phi_{f} S_{f}^{\alpha}\left(., \gamma p_{i n i}\right) \gamma \bar{\psi}(\mathbf{x}, 0) d \tau_{f}(\mathbf{x}) d t .
$$

Diffusion terms. From Lemma 5.1 in the Appendix, Proposition 2.1, Lemma 3.6 and hypothesis $\left(\mathrm{H}_{4}\right)$, we deduce that

$$
\sum_{n=1}^{N} \Delta t^{n} B_{\mathcal{D}_{m}}^{\alpha, n}\left(\psi\left(t^{n-1}\right)\right) \rightarrow \int_{0}^{T} \int_{\Omega} k_{m}^{\alpha}\left(\mathbf{x}, S_{m}^{\alpha}(., \bar{p})\right) \Lambda_{m} \nabla \bar{u} \cdot \nabla \bar{\psi} d \mathbf{x}
$$

and

$$
\sum_{n=1}^{N} \Delta t^{n} B_{\mathcal{D}_{f}}^{\alpha, n}\left(\psi\left(t^{n-1}\right)\right) \rightarrow \int_{0}^{T} \int_{\Gamma} k_{f}^{\alpha}\left(\mathbf{x}, S_{f}^{\alpha}(., \bar{p})\right) \Lambda_{f} \nabla_{\tau} \gamma \bar{u} \cdot \nabla_{\tau} \gamma \bar{\psi} d \tau_{f}(\mathbf{x}) .
$$

Source terms. From Lemma 2.2 and Proposition 2.1, we deduce that

$$
\sum_{n=1}^{N} \Delta t^{n} C_{\mathcal{D}_{m}}^{\alpha, n}\left(\psi\left(t^{n-1}\right)\right)=\sum_{n=1}^{N} \int_{t^{n-1}}^{t^{n}} \int_{\Omega} h_{m}^{\alpha} \pi_{\mathcal{D}_{m}} \psi\left(t^{n-1}\right) d \mathbf{x} d t \rightarrow \int_{0}^{T} \int_{\Omega} h_{m}^{\alpha} \bar{\psi} d \mathbf{x} d t
$$

and

$$
\sum_{n=1}^{N} \Delta t^{n} C_{\mathcal{D}_{f}}^{\alpha, n}\left(\psi\left(t^{n-1}\right)\right)=\sum_{n=1}^{N} \int_{t^{n-1}}^{t^{n}} \int_{\Gamma} h_{f}^{\alpha} \pi_{\mathcal{D}_{f}} \psi\left(t^{n-1}\right) d \tau_{f}(\mathbf{x}) d t \rightarrow \int_{0}^{T} \int_{\Gamma} h_{f}^{\alpha} \gamma \bar{\psi} d \tau_{f}(\mathbf{x}) d t
$$




\section{Numerical experiments}

The implementation of the VAG scheme is based on a flux formulation with upwinding of the mobilities rather than the discrete variational formulation (4) in order to improve the stability of the solution on coarse meshes for convective dominant regimes. For a given $u \in X_{\mathcal{D}}$, the definition of the fluxes follows the same ideas as in [15], [6]. The matrix fluxes connect the cell $K \in \mathcal{M}$ to its vertices or fracture faces $\nu \in \Xi_{K}=\mathcal{V}_{K} \cup\left(\mathcal{F}_{\Gamma} \cap \mathcal{F}_{K}\right)$ :

$$
F_{K, \nu}(u)=-\int_{K} \Lambda_{m}(\mathbf{x}) \nabla \pi_{\mathcal{T}} u(\mathbf{x}) \cdot \nabla \eta_{\nu}(\mathbf{x}) d \mathbf{x}=\sum_{\nu^{\prime} \in \Xi_{K}} a_{K, \nu}^{\nu^{\prime}}\left(u_{K}-u_{\nu^{\prime}}\right)
$$

with $a_{K, \nu}^{\nu^{\prime}}=\int_{K} \Lambda(\mathbf{x}) \nabla \eta_{\nu}(\mathbf{x}) \cdot \nabla \eta_{\nu^{\prime}}(\mathbf{x}) d \mathbf{x}$. The fracture fluxes connect the face $\sigma \in \mathcal{F}_{\Gamma}$ to its vertices $\mathbf{s} \in \mathcal{V}_{\sigma}:$

$$
F_{\sigma, \mathbf{s}}(u)=-\int_{\sigma} \Lambda_{f}(\mathbf{x}) \nabla_{\tau} \gamma \pi_{\mathcal{T}} u(\mathbf{x}) \cdot \nabla_{\tau} \gamma \eta_{\mathbf{s}}(\mathbf{x}) d \tau_{f}(\mathbf{x})=\sum_{\mathbf{s}^{\prime} \in \mathcal{V}_{\sigma}} a_{\sigma, \mathbf{s}}^{\mathbf{s}^{\prime}}\left(u_{\sigma}-u_{\mathbf{s}^{\prime}}\right)
$$

with $a_{\sigma, \mathbf{s}}^{\mathbf{s}^{\prime}}=\int_{\sigma} \Lambda_{f}(\mathbf{x}) \nabla_{\tau} \gamma \eta_{\mathbf{s}}(\mathbf{x}) \cdot \nabla_{\tau} \gamma \eta_{\mathbf{s}^{\prime}}(\mathbf{x}) d \tau_{f}(\mathbf{x})$.

Given cellwise constant rocktypes in the matrix and facewise constant rocktypes in the fracture network, let us define for $\alpha=1,2$

$$
S_{K}^{\alpha}=S_{m}^{\alpha}\left(\mathbf{x}_{K}, p_{K}\right), K \in \mathcal{M}, \quad S_{K, \nu}^{\alpha}=S_{m}^{\alpha}\left(\mathbf{x}_{K}, p_{\nu}\right), K \in \mathcal{M}, \nu \in \Xi_{K},
$$

and

$$
S_{\sigma}^{\alpha}=S_{f}^{\alpha}\left(\mathbf{x}_{\sigma}, p_{\sigma}\right), \sigma \in \mathcal{F}_{\Gamma}, \quad S_{\sigma, \mathbf{s}}^{\alpha}=S_{f}^{\alpha}\left(\mathbf{x}_{\sigma}, p_{\mathbf{s}}\right), \sigma \in \mathcal{F}_{\Gamma}, \mathbf{s} \in \mathcal{V}_{\sigma} .
$$

Let us set $\phi_{K}=\int_{K} \phi_{m}(\mathbf{x}) d \mathbf{x}$ and $\phi_{\sigma}=\int_{\sigma} \phi_{f}(\mathbf{x}) d \tau_{f}(\mathbf{x})$. Given $p^{0} \in X_{\mathcal{D}}^{0}$, the VAG upwind scheme looks for $u^{1, n} \in X_{\mathcal{D}}^{0}, u^{2, n} \in X_{\mathcal{D}}^{0}, n=1, \cdots, N$, such that for all $\alpha=1,2$ and for all $v \in X_{\mathcal{D}}^{0}$ one has:

$$
\left\{\begin{array}{l}
\sum_{K \in \mathcal{M}}\left(\frac{\phi_{K}}{\Delta t^{n}}\left(1-\sum_{\nu \in \Xi_{K} \cap \mathcal{V}_{i n t}} \alpha_{K, \nu}\right)\left(S_{K}^{\alpha, n}-S_{K}^{\alpha, n-1}\right)+\sum_{\nu \in \Xi_{K}} k^{\alpha}\left(\mathbf{x}_{K}, S_{K, \nu, u p}^{\alpha, n}\right) F_{K, \nu}\left(u^{\alpha, n}\right)\right) v_{K} \\
+\sum_{K \in \mathcal{M}} \sum_{\nu \in \Xi_{K} \cap \mathcal{V}_{i n t}}\left(\frac{\phi_{K}}{\Delta t^{n}} \alpha_{K, \nu}\left(S_{K, \nu}^{\alpha, n}-S_{K, \nu}^{\alpha, n-1}\right)-k^{\alpha}\left(\mathbf{x}_{K}, S_{K, \nu, u p}^{\alpha, n}\right) F_{K, \nu}\left(u^{\alpha, n}\right)\right) v_{\nu} \\
+\sum_{\sigma \in \mathcal{F}_{\Gamma}}\left(\frac{\phi_{\sigma}}{\Delta t^{n}}\left(1-\sum_{\mathbf{s} \in \mathcal{V}_{\sigma} \cap \mathcal{V}_{i n t}} \alpha_{\sigma, \mathbf{s}}\right)\left(S_{\sigma}^{\alpha, n}-S_{\sigma}^{\alpha, n-1}\right)+\sum_{\mathbf{s} \in \mathcal{V}_{\sigma}} k_{f}^{\alpha}\left(\mathbf{x}_{\sigma}, S_{\sigma, \mathbf{s}, u p}^{\alpha, n}\right) F_{\sigma, \mathbf{s}}\left(u^{\alpha, n}\right)\right) v_{\sigma} \\
+\sum_{\sigma \in \mathcal{F}_{\Gamma}} \sum_{\mathbf{s} \in \mathcal{V}_{\sigma} \cap \mathcal{V}_{i n t}}\left(\frac{\phi_{\sigma}}{\Delta t^{n}} \alpha_{\sigma, \mathbf{s}}\left(S_{\sigma, \mathbf{s}}^{\alpha, n}-S_{\sigma, \mathbf{s}}^{\alpha, n-1}\right)-k_{f}^{\alpha}\left(\mathbf{x}_{\sigma}, S_{\sigma, \mathbf{s}, u p}^{\alpha, n}\right) F_{\sigma, \mathbf{s}}\left(u^{\alpha, n}\right)\right) v_{\mathbf{s}}=0,
\end{array}\right.
$$

with the upwinding

$$
\left\{\begin{array} { l } 
{ S _ { K , \nu , u p } ^ { \alpha , n } = S _ { K } ^ { \alpha , n } \text { if } F _ { K , \nu } ( u ^ { \alpha , n } ) \geq 0 , } \\
{ S _ { K , \nu , u p } ^ { \alpha , n } = S _ { K , \nu } ^ { \alpha , n } \text { if } F _ { K , \nu } ( u ^ { \alpha , n } ) < 0 , }
\end{array} \quad \left\{\begin{array}{l}
S_{\sigma, n}^{\alpha, n}, u p=S_{\sigma}^{\alpha, n} \text { if } F_{\sigma, \mathbf{s}}\left(u^{\alpha, n}\right) \geq 0 \\
S_{\sigma, \mathbf{s}, u p}^{\alpha, n}=S_{\sigma, \mathbf{s}}^{\alpha, n} \text { if } F_{\sigma, \mathbf{s}}\left(u^{\alpha, n}\right)<0 .
\end{array}\right.\right.
$$

In the following numerical experiments, the volume fractions $\alpha_{K, \nu}, \alpha_{\sigma, \mathbf{s}}$ are chosen to avoid the mixing of the fracture and matrix rocktypes. They are such that $\alpha_{K, \nu}=0$ if $\nu \in \Xi_{K}$ belongs to the fracture network $\Gamma, \alpha_{K, \nu}=\omega_{m}$ otherwise, and such that $\alpha_{\sigma, \mathbf{s}}=\omega_{f}$ for $\mathbf{s} \in \mathcal{V}_{\sigma}$.

To illustrate the importance of non mixing rocktypes with large permeability contrasts, this choice denoted by VAG-1 will be compared with a second choice denoted by VAG-2 for which we simply set $\alpha_{K, \nu}=\omega_{m}$ and $\alpha_{\sigma, \mathbf{s}}=\omega_{f}$ for all $\nu \in \Xi_{K}, \mathbf{s} \in \mathcal{V}_{\sigma}$. In order to roughly balance the volumes, the parameters $\omega_{m}$ and $\omega_{f}$ are set in the following tests to $\omega_{m}=0.15$ in $2 \mathrm{D}$ and 0.05 in $3 \mathrm{D}$, and to $\omega_{f}=0.25$ in $2 \mathrm{D}$ and 0.1 in $3 \mathrm{D}$. Figure 3 exhibits an example of the control volumes at cells, fracture face and nodes for both the VAG-1 and VAG-2 choices. 


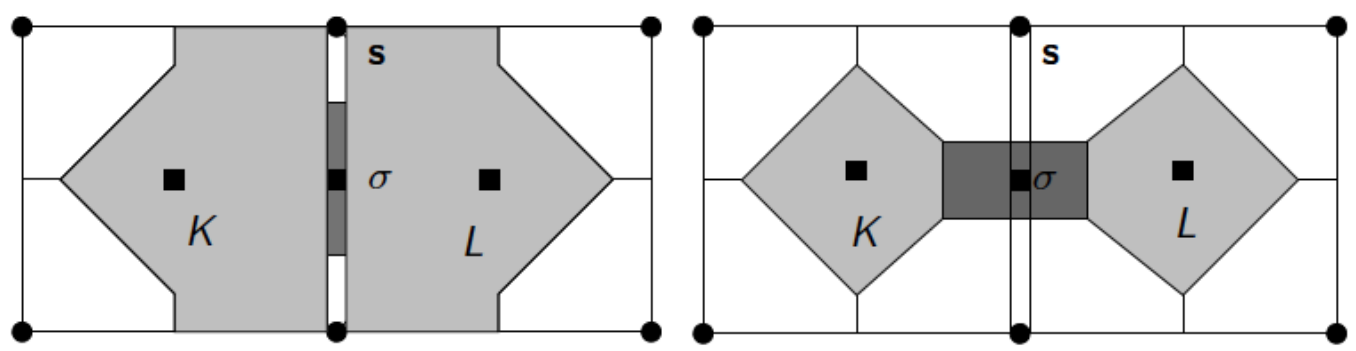

Figure 3: For VAG-1 (left) and VAG-2 (right), example of the choices of the control volumes at cells, fracture face, and nodes, in the case of two cells $K$ and $L$ splitted by one fracture face $\sigma$ (the width of the fracture has been enlarged in this figure).

The nonlinear systems obtained at each time step are solved by a Newton Raphson algorithm. The time stepping is defined by an initial time step, a maximum time step and the following rule: if the Newton solver does not converge after 20 iterations, the time step is chopped by a factor 2 and recomputed. The time step is increased by a factor 1.2 after each successful time step until it reaches the maximum time step. The stopping criteria on the relative residuals are fixed to $10^{-7}$ for the GMRes solver and to $10^{-6}$ for the Newton solver. A CPR-AMG right preconditioner [25], [30] is used in the GMRes iterative solver. Let us also stress that, using the two equations in each cell, the cell unknowns are eliminated from the discrete linearized system at each Newton iteration without any fill-in, reducing the Jacobian system to nodal and fracture face unknowns only. Note that the above discretization is readily extended to take into account gravity using the fluxes

$$
F_{K, \nu}^{\alpha}\left(u^{\alpha}\right)=F_{K, \nu}\left(u^{\alpha}\right)+\rho^{\alpha} g F_{K, \nu}(z), \quad F_{\sigma, \mathbf{s}}^{\alpha}\left(u^{\alpha}\right)=F_{\sigma, \mathbf{s}}\left(u^{\alpha}\right)+\rho^{\alpha} g F_{\sigma, \mathbf{s}}(z),
$$

where $g$ is the gravitational constant, $\rho^{\alpha}$ is the density of the phase $\alpha$, and $z=\left(z_{K}, K \in \mathcal{M}, z_{\sigma}, \sigma \in\right.$ $\left.\mathcal{F}_{\Gamma}, z_{\mathbf{s}}, \mathbf{s} \in \mathcal{V}\right)$. The upwinding of the mobilities is defined with respect to these new fluxes.

In the following test cases, the inverse of the capillary pressure monotone graph will be defined by the Corey law

$$
S_{j}^{1}(p)= \begin{cases}0 & \text { if } p<a_{j}, \\ \left(1-s_{r, j}^{2}\right)\left(1-e^{\frac{a_{j}-p}{b_{j}}}\right) & \text { if } p \geq a_{j},\end{cases}
$$

and the mobilities of the two phases are given by the Corey laws

$$
k_{j}^{\alpha}\left(\mathbf{x}, s^{\alpha}\right)= \begin{cases}0 & \text { if } \bar{s}^{\alpha}<0, \\ \frac{1}{\mu^{\alpha}} & \text { if } \bar{s}^{\alpha}>1, \\ \frac{\left(\bar{s}^{\alpha}\right)^{2}}{\mu^{\alpha}} & \text { else, }\end{cases}
$$

for phase $\alpha=1$ (oil), and phase $\alpha=2$ (water) where $\bar{s}^{1}=\frac{s^{1}-s_{r, j}^{1}}{1-s_{r, j}^{1}-s_{r, j}^{2}}$, and $\bar{s}^{2}=\frac{s^{2}-s_{r, j}^{2}}{1-s_{r, j}^{2}-s_{r, j}^{1}}$ are the reduced saturations, $s_{r, j}^{1}, s_{r, j}^{2}$ are the residual saturations for both phases, $a_{j}$ is the entry pressure, and $\mu^{1}, \mu^{2}$ are the viscosities of the phases.

\subsection{Oil migration in a $2 \mathrm{D}$ basin with one barrier and a fault}

We consider the simulation of the oil migration process, within the $2 \mathrm{D}$ cross section $\Omega=(0, L) \times(0, H)$ of a basin with $H=L=100 \mathrm{~m}$ (see Figure 4). Let us denote by $(x, y)$ the Cartesian coordinates of $\mathbf{x}$ and let us define the two points $\mathbf{x}_{1}=(50,50)$ and $\mathbf{x}_{2}=(50,69.177)$. The basin includes an immersed fault $\Gamma=\left(\mathbf{x}_{1}, \mathbf{x}_{2}\right)$, a barrier $\Omega_{2}=\left\{(x, y) \in \Omega \mid 25+\frac{x}{2}<y<35+\frac{x}{2}, x \in(0,1)\right\} \backslash \bar{\Gamma}$, and a drain $\Omega_{1}=\Omega \backslash\left(\bar{\Omega}_{2} \cup \bar{\Gamma}\right)$. The permeability $\Lambda_{m}(\mathbf{x})$ is defined by the tensor

$$
\Lambda_{m}(\mathbf{x})=\lambda_{1}\left(\begin{array}{ll}
0.82 & -0.36 \\
-0.36 & 0.28
\end{array}\right) \text {. }
$$


for $\mathbf{x} \in \Omega_{1}$, and by $\Lambda_{m}(\mathbf{x})=\frac{\lambda_{1}}{100} \operatorname{Id}$ for $\mathbf{x} \in \Omega_{2}$, with $\lambda_{1}=10^{-12} \mathrm{~m}^{2}$. Note that the eigenvalues of $\Lambda_{m}(\mathbf{x})$ for $\mathbf{x} \in \Omega_{1}$ are $0.1 \lambda_{1}$ and $\lambda_{1}$ and the corresponding eigenvectors are $\left(\frac{1}{\sqrt{5}}, \frac{2}{\sqrt{5}}\right),\left(\frac{2}{\sqrt{5}},-\frac{1}{\sqrt{5}}\right)$.

The permeability of the fault $\Gamma$ is defined by $\Lambda_{f}=100 \lambda_{1} \mathrm{Id}$ and its width is equal to $d_{f}=0.1 \mathrm{~m}$. The porosity is constant and equal to $\phi_{m}=\phi_{f}=0.1$.

The inverse of the capillary pressure monotone graph in each subdomain $\Omega_{j}, j=1,2$ is exhibited in figure 4 and defined by (12) with the parameters $a_{1}=10^{5} \mathrm{~Pa}, a_{2}=210^{5} \mathrm{~Pa}, b_{1}=10^{2} \mathrm{~Pa}$, and $b_{2}=10^{4} \mathrm{~Pa}, s_{r, 1}^{2}=s_{r, 2}^{2}=0$. In the matrix $S_{m}^{1}(\mathbf{x}, p)$ is equal to $S_{1}^{1}(p)$ for $\mathbf{x} \in \Omega_{1}$, and to $S_{2}^{1}(p)$ for $\mathbf{x} \in \Omega_{2}$. In the fault, the inverse of the capillary pressure is defined by $S_{f}^{1}(\mathbf{x}, p)=S_{1}^{1}(p)$ for $\mathbf{x} \in \Gamma$. The mobilities of the two phases are given by (13) with $\mu^{1}=0.005$ Pa.s and $\mu^{2}=0.001$ Pa.s and $s_{r}^{1}=s_{r}^{2}=0$ both in the matrix and in the fracture.

The migration occurs by gravity due to the lower density of the oil phase $\rho^{1}=850 \mathrm{Kg} / \mathrm{m}^{3}$ compared with the water phase $\rho^{2}=1000 \mathrm{Kg} / \mathrm{m}^{3}$. Phase 1 is injected at the bottom boundary $(0,10) \times\{0\}$ with imposed pressures $u^{2}=810^{6}+\rho^{2} g H \mathrm{~Pa}, u^{1}=u^{2}+\left(S_{1}^{1}\right)^{-1}(0.8)$ corresponding to an input phase 1 saturation $s^{1}=0.8$. At the top boundary the phase pressures are fixed to $u^{2}(\mathbf{x})=810^{6}$, and $u^{1}(\mathbf{x})=u^{2}(\mathbf{x})+a_{1}$. The remaining boundaries are assumed to be impervious as well as the boundaries of the fault. At initial time the porous media is saturated with phase 2 with a hydrostatic pressure $u_{i n i}^{2}(\mathbf{x})=810^{6}+\rho_{2} g(H-y)$, and a phase 1 pressure defined by $u_{i n i}^{1}(\mathbf{x})=u_{i n i}^{2}(\mathbf{x})+a_{j}$ for $\mathbf{x} \in \Omega_{j}$, $j=1,2$, and $u_{i n i}^{1}(\mathbf{x})=u_{i n i}^{2}(\mathbf{x})+a_{1}$ for $\mathbf{x} \in \Gamma$.

The mesh is a $n_{x} \times n_{x}$ topologically Cartesian quadrangular grid which is refined below the barrier as exhibited in figure 4 . The simulation is done over 1800 days with an initial time step equal to the maximum time step and fixed to 5 days. The VAG-1 choice of the control volumes is fixed for all simulations of this test case.

Figure 5 exhibits the oil (phase 1) saturation at final time. We clearly see that the oil phase rises by gravity along the direction of the highest permeability and accumulates below the barrier. Due to the saturation jump condition at the barrier drain interface given by the capillary pressure functions, oil can only cross the barrier through the fault. Figure 5 exhibits the convergence of the oil saturation $s^{1}$ at final time for the family of meshes obtained with $n_{x}=50,100,200,400$. Figure 6 plots the volume of oil below the barrier, above the barrier, and in the fault function of time for this family of meshes. In both cases, we observe the numerical convergence of the solution when the mesh is refined.

Table 4.1 exhibits the numerical behavior of the simulation for the family of quadrangular meshes with a rather good scalability both in terms of linear and nonlinear solvers.

\begin{tabular}{|c|c|c|c|c|c|}
\hline Mesh & $N_{\Delta t}$ & $N_{\text {Chop }}$ & $N_{\text {Newton }}$ & $N_{\text {GMRes }}$ & CPU (s) \\
\hline $50 \times 50$ & 360 & 0 & 2.11 & 9.35 & 22.20 \\
\hline $100 \times 100$ & 364 & 2 & 2.34 & 11.75 & 106 \\
\hline $200 \times 200$ & 369 & 5 & 3.37 & 14.45 & 637 \\
\hline $400 \times 400$ & 392 & 17 & 4.41 & 17.84 & 3933 \\
\hline
\end{tabular}

Table 1: For each mesh: number $N_{\Delta t}$ of successful time steps, number $N_{C h o p}$ of time step chops, number $N_{\text {Newton }}$ of Newton iterations per successful time step, number $N_{\text {GMRes }}$ of GMRes iterations by Newton iteration, CPU time in seconds. 

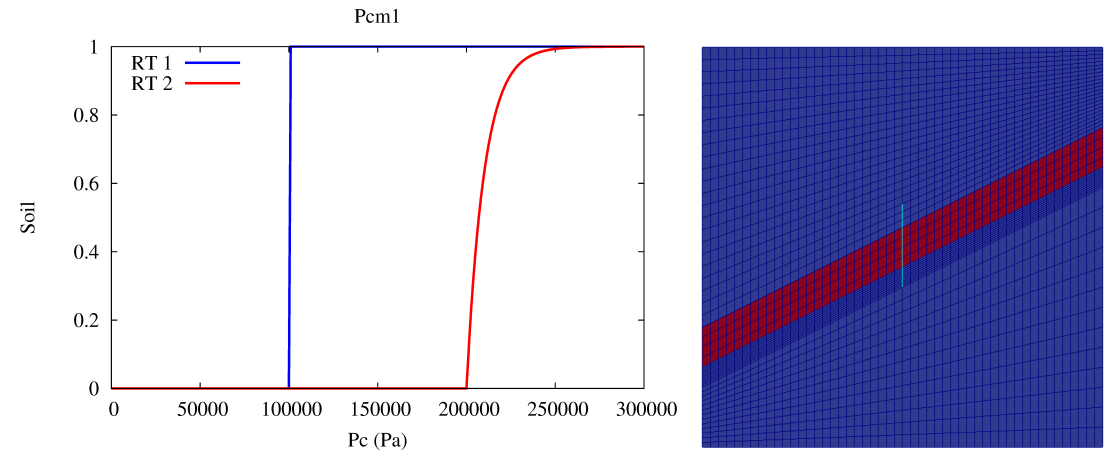

Figure 4: Inverses of the extended monotone graphs of the capillary pressures $S_{1}^{1}$ (in blue in subdomain $\Omega_{1}$ and in the fault $\Gamma_{f}$ ) and $S_{2}^{1}$ (in red in subdomain $\Omega_{2}$ ). Mesh of the basin with the barrier in red, the drain in blue, and the fault in cyan.
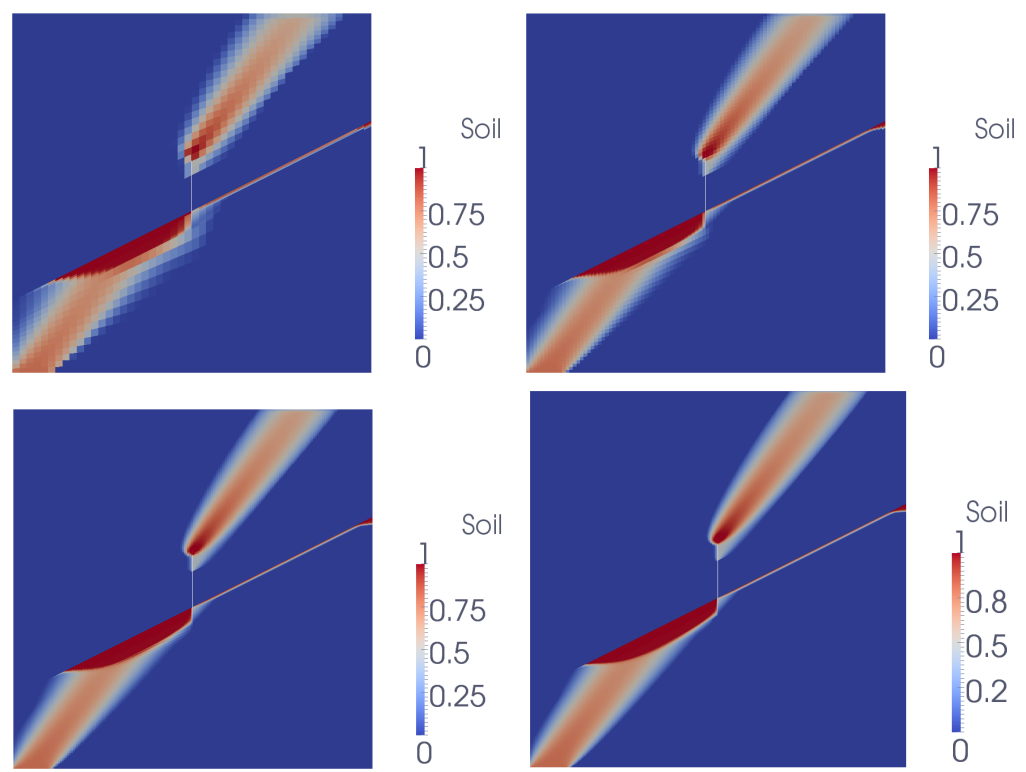

Figure 5: Discrete oil saturations at final time obtained for $n_{x}=50,100,200,400$. 

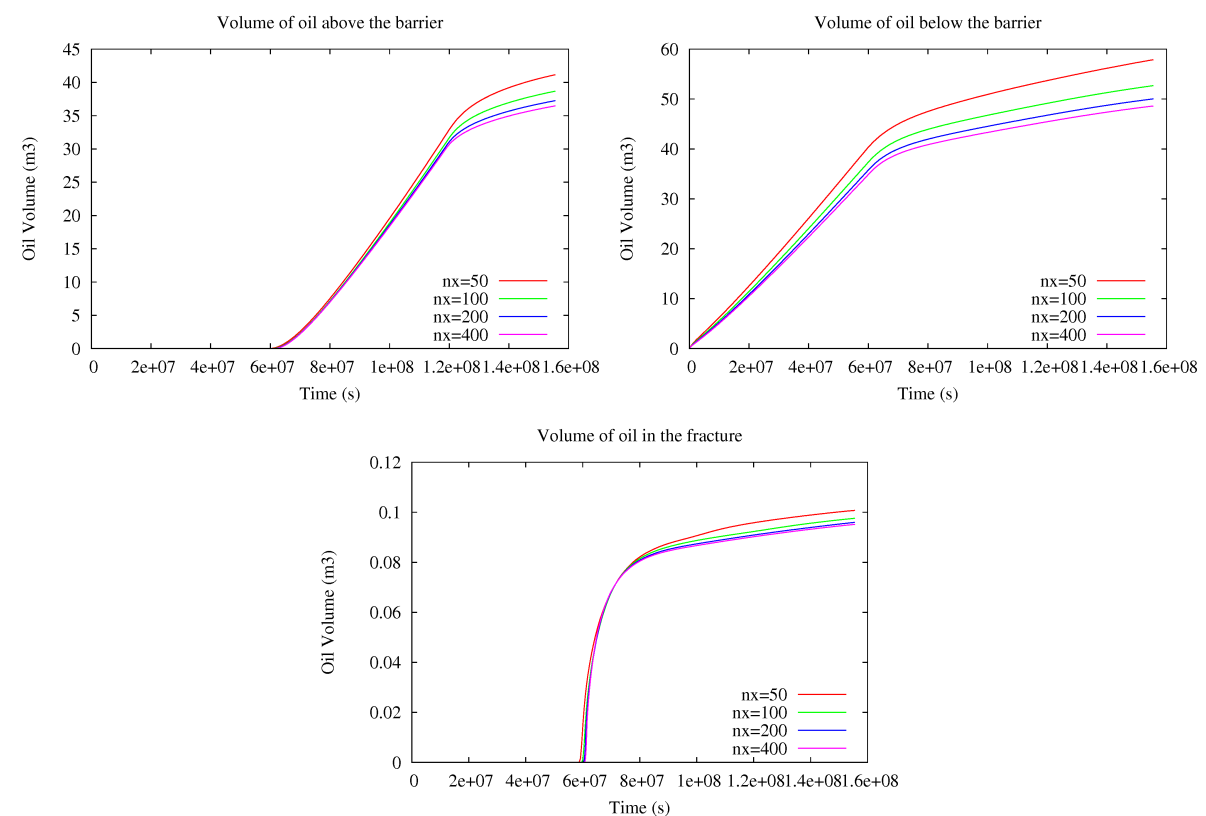

Figure 6: Volume of oil above the barrier, below the barrier and in the fault function of time for $n_{x}=50,100,200,400$.

\subsection{Oil migration in a basin with a random network of fractures}

We consider the migration of oil in the $2 \mathrm{D}$ cross section $\Omega=(0, L) \times(0, H)$ of a basin with $H=L=100$ $\mathrm{m}$. The basin comprises a random network of 927 fractures exhibited in Figure 7 . The permeability of the matrix $\Lambda_{m}=\lambda_{m}$ Id and the permeability of the fractures $\Lambda_{f}=\lambda_{f}$ Id are highly contrasted with $\lambda_{m}=10^{-15} \mathrm{~m}^{2}, \lambda_{f}=10^{-10} \mathrm{~m}^{2}$. The width of the fractures is fixed to $d_{f}=0.01 \mathrm{~m}$ and their porosity to $\phi_{f}=0.3$. The porosity of the matrix is set to $\phi_{m}=0.1$.

The inverses of the capillary pressure monotone graph in the matrix $(j=m)$ and in the fractures $(j=f)$ are exhibited in Figure 7 and defined by the Corey law (12) with the rocktype $b_{m}=510^{3} \mathrm{~Pa}$, $s_{r, m}^{2}=0.2, s_{r, m}^{1}=0$ in the matrix and the rocktype $b_{f}=10^{2} \mathrm{~Pa}, s_{r, f}^{2}=s_{r, f}^{1}=0$ in the fractures. The mobilities are defined for $j=m$ and $j=f$ by the Corey law (13) with $\mu^{1}=0.005$ Pa.s and $\mu^{2}=0.001$ Pa.s.

The densities of phases are fixed to $\rho^{1}=700 \mathrm{Kg} / \mathrm{m}^{3}$ for the oil phase and $\rho^{2}=1000 \mathrm{Kg} / \mathrm{m}^{3}$ for the water phase.

Phase 1 is injected at the bottom boundary $(25,75) \times\{0\}$ with imposed pressures $u^{2}(\mathbf{x})=8.110^{6}+$ $\rho^{2} g H \mathrm{~Pa}, u^{1}(\mathbf{x})=u^{2}(\mathbf{x})+\left(S_{f}^{1}\right)^{-1}(0.999999)$ corresponding to an input phase 1 saturation $s^{1}=$ 0.999999 in the fractures. At the top boundary, the phase pressures are fixed to $u^{2}(\mathbf{x})=810^{6}$ and $u^{1}(\mathbf{x})=u^{2}(\mathbf{x})$. The remaining boundaries of the basin are assumed to be impervious. The boundaries of the fracture network not located at the top boundary of the basin nor at the bottom boundary $(25,75) \times\{0\}$ are also assumed impervious.

At initial time the porous media is saturated with phase 2 with a hydrostatic pressure $u_{i n i}^{2}(\mathbf{x})=$ $810^{6}+\rho_{2} g(H-y)$, and a phase 1 pressure defined by $u_{i n i}^{1}(\mathbf{x})=u_{i n i}^{2}(\mathbf{x})$.

The mesh is a $n_{x} \times n_{x}$ topologically Cartesian quadrangular grid obtained by a uniform refinement of a given randomly distorted $30 \times 30$ Cartesian grid (see figure 7 ). The simulation is fixed to 75 days with an initial time step of 0.01 day and a maximum time step of 0.1 day. All the runs for this test case are performed on a laptop equipped with a dual core $3 \mathrm{GHz}$.

Figure 8 exhibits the discrete oil saturation at final time obtained with a topologically Cartesian mesh of size $n_{x}=480$, and a non uniform refinement of the mesh with cell widths away from the 
fracture equal to roughly $2.6,4.1,6.45,10.1,16.0,25.2,39.6,62.4 \mathrm{~cm}$ (to be compared with the width of say $21 \mathrm{~cm}$ obtained with the uniform mesh for $n_{x}=480$ ). The choice of the volume fractions $\alpha_{K, \nu}$, $\alpha_{\sigma, \mathbf{s}}$ is set to VAG-1. The numerical convergence of this solution has been checked and it will be our reference solution for the comparison of the VAG-1 and VAG-2 choices. The Figures 9, 10 compare the convergence of the oil saturation on the family of uniformly refined meshes with $n_{x}=60,120,240,480$, for the two choices of the volume fractions. It is clear that the choice VAG-1 which avoids to mix matrix and fracture volumes provides a much better convergence. It is explained in Figure 11 exhibiting that the choice VAG-2 of the volume distribution yields a too large volume of oil in the matrix and a too small volume of oil in the fractures due to the enlarged volumes at the matrix fracture interfaces. This is a clear advantage of the VAG scheme compared with usual CVFE approaches which cannot avoid the mixing of fracture and matrix volumes due to the use of a dual mesh and the absence of cell unknowns.

The numerical behavior of the simulations for both choices of the distribution of the volumes and for the family of meshes is exhibited in Table 4.2 showing the number of successful time steps, the number of time step chops, the number of Newton iterations by successful time steps, the number of GMRes iterations by Newton iteration, the CPU time in seconds, and the maximum CFL number. This maximum CFL number is the one obtained for the oil saturation Buckley Leverett equation (without the capillary diffusion) discretized by an upwind monotone scheme and an Euler explicit time integration, and using the total Darcy velocity and the buoyancy forces of the simulation.

In both cases a rather good scalability is obtained both in terms of nonlinear and linear solvers although very large CFL numbers are observed in the fracture network. The results are slightly better for VAG-2 due to the larger control volumes at the matrix fracture interfaces as can be checked on the comparison of the maximum CFL numbers in table 4.2.
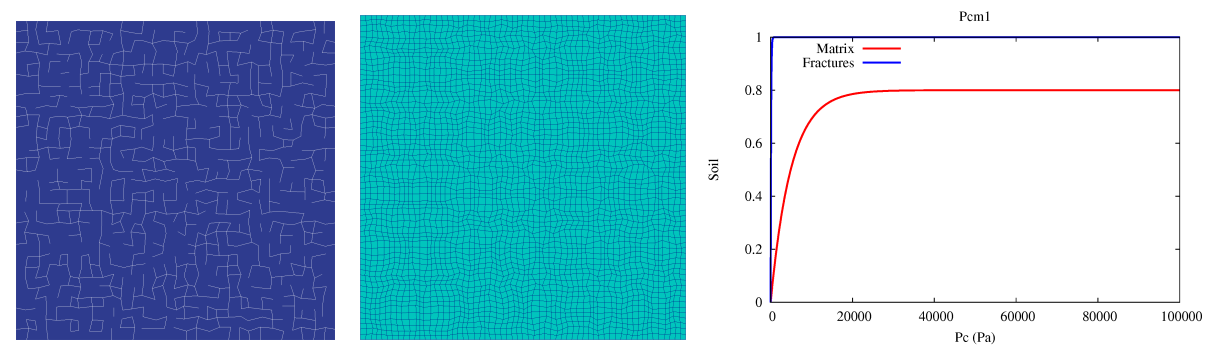

Figure 7: Network of 927 fractures and mesh obtained for $n_{x}=60$. Inverses of the extended monotone graphs of the capillary pressures $S_{m}^{1}$ in the matrix, and $S_{f}^{1}$ in the fractures.

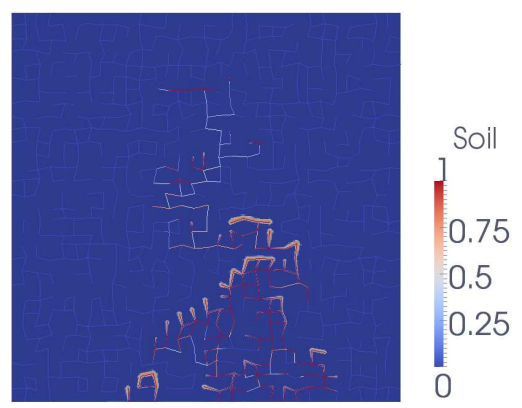

Figure 8: Discrete oil saturation at final time obtained for $n_{x}=480$ with refinement at the matrix fracture interfaces and the choice VAG-1 of the volumes. 

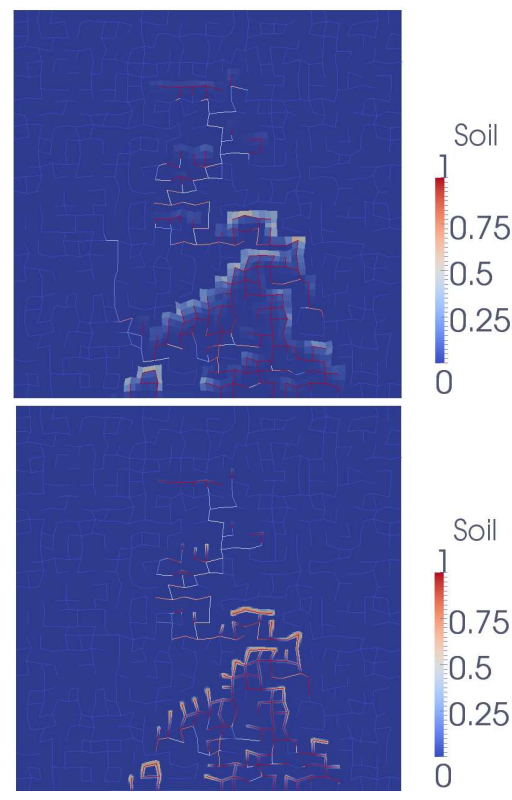

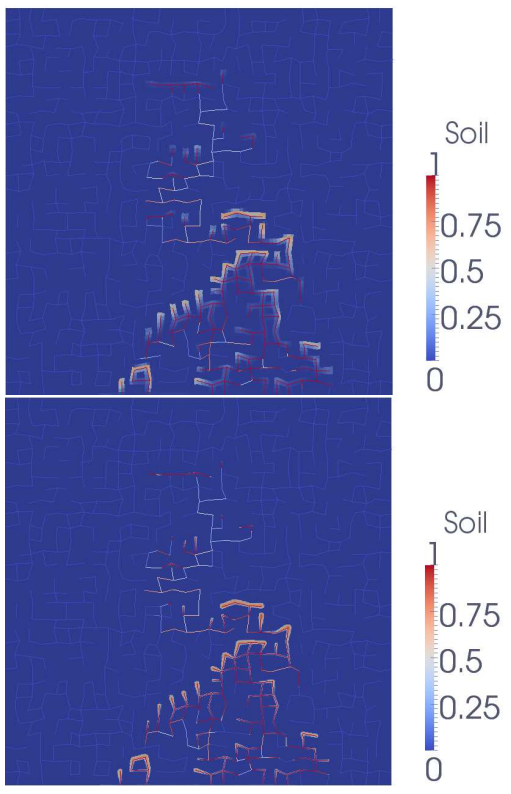

Figure 9: Discrete oil saturation at final time obtained for the family of uniformly refined meshes $n_{x}=60,120,240,480$, and the choice VAG-1 of the volumes.
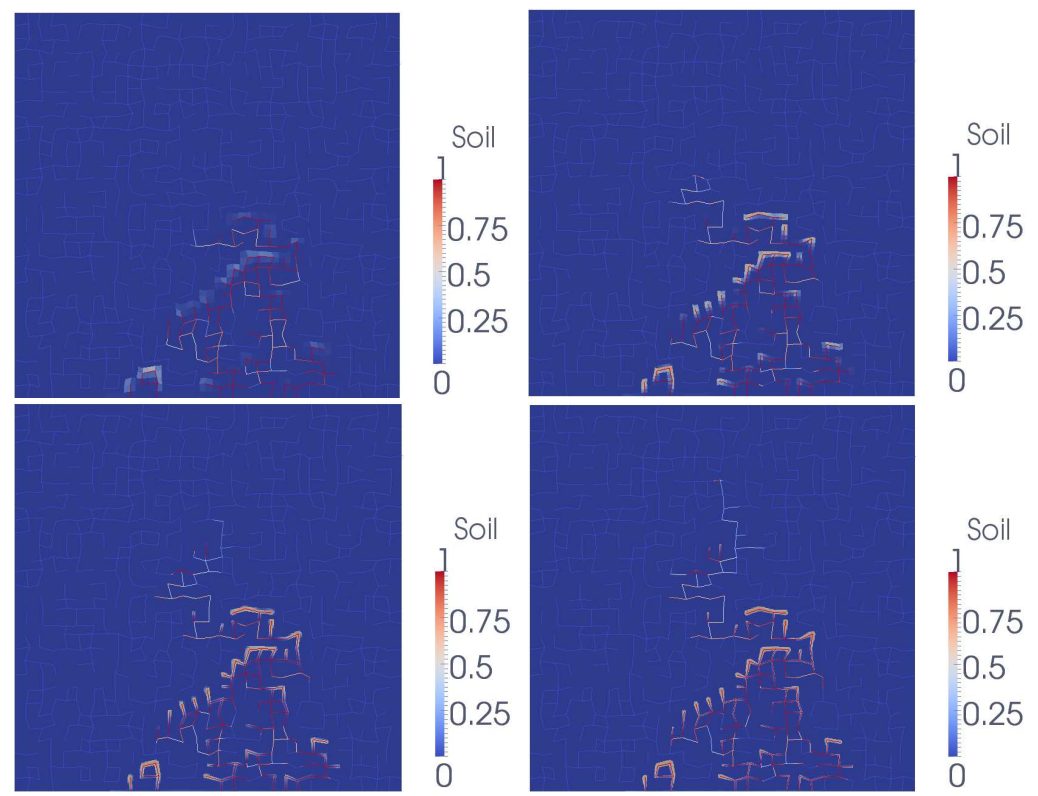

Figure 10: Discrete oil saturation at final time obtained for the family of uniformly refined meshes $n_{x}=60,120,240,480$, and the choice VAG-2 of the volumes. 

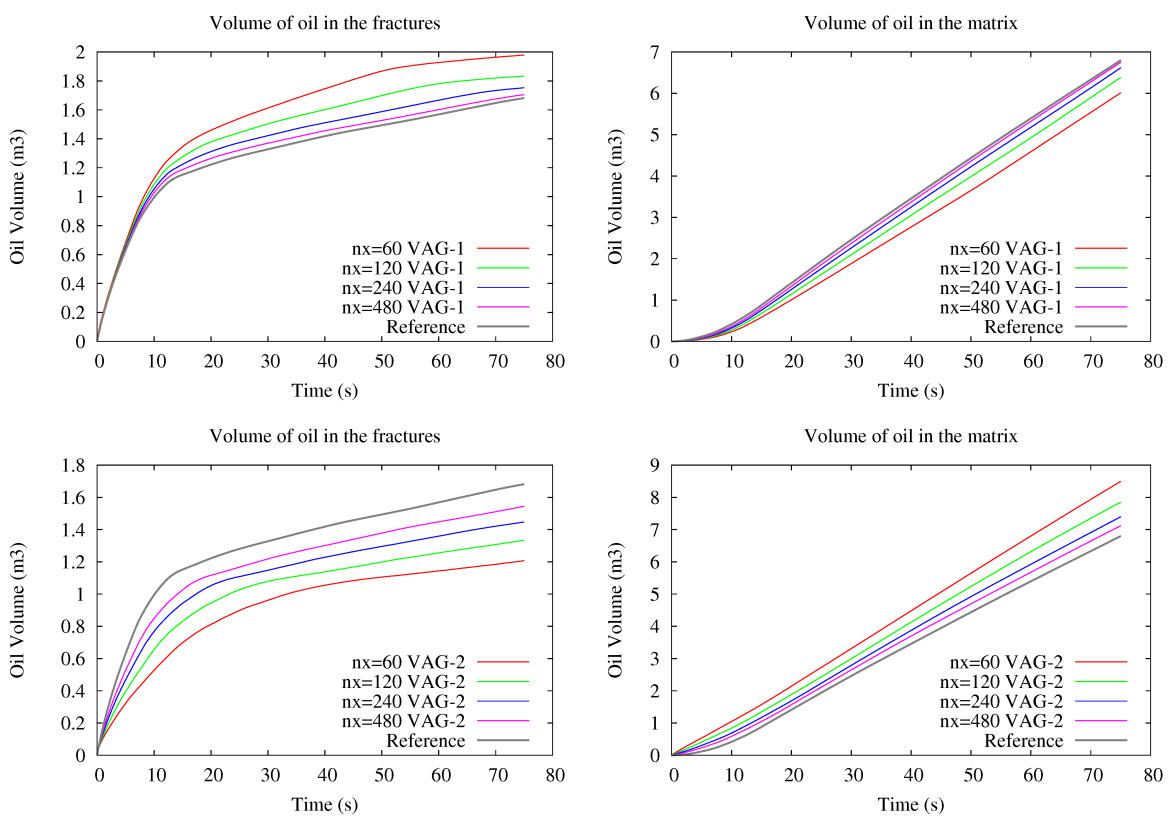

Figure 11: Volume of oil in the fracture and in the matrix function of time for $n_{x}=60,120,240,480$, and for the two choices VAG-1 and VAG-2 of the distribution of the volumes.

\begin{tabular}{|c|c|c|c|c|c|c|c|}
\hline Volumes & $n_{x}$ & $N_{\Delta t}$ & $N_{\text {Chop }}$ & $N_{\text {Newton }}$ & $N_{\text {GMRes }}$ & CPU (s) & CFL \\
\hline VAG-1 & 60 & 759 & 0 & 2.62 & 8.08 & 164 & 210 \\
\hline VAG-1 & 120 & 759 & 0 & 2.90 & 8.84 & 535 & 420 \\
\hline VAG-1 & 240 & 777 & 14 & 3.94 & 9.44 & 2480 & 840 \\
\hline VAG-1 & 480 & 889 & 69 & 5.79 & 9.85 & 15062 & 1675 \\
\hline \hline VAG-2 & 60 & 759 & 0 & 2.08 & 6.26 & 126 & 6.4 \\
\hline VAG-2 & 120 & 759 & 0 & 2.48 & 6.81 & 441 & 27 \\
\hline VAG-2 & 240 & 759 & 0 & 3.16 & 7.83 & 1868 & 106 \\
\hline VAG-2 & 480 & 788 & 21 & 4.98 & 8.53 & 11074 & 384 \\
\hline
\end{tabular}

Table 2: For each choice VAG-1 and VAG-2 of the volume distribution and each mesh size $n_{x}=$ 60,120, 240,480: number $N_{\Delta t}$ of successful time steps, number $N_{C h o p}$ of time step chops, number $N_{\text {Newton }}$ of Newton iterations per successful time step, number $N_{\text {GMRes }}$ of GMRes iterations by Newton iteration, CPU time in seconds, maximum CFL number.

\subsection{D network of fractures}

This test case considers the migration of oil in a 3D basin $\Omega=(0, L) \times(0, L) \times(0, H)$ with $H=L=100$ $\mathrm{m}$. Figure 12 exhibits the test case geometry where the fractures in the cube are represented by parallelograms and are intersected with the top and the bottom of the basin. The family of tetrahedral meshes is generated using TetGen [32] in order to be refined at the neighbourhood of the fracture network. Figure 12 shows the coarsest mesh $i_{\text {mesh }}=1$, and Table 3 defines for each mesh the number $N b_{\text {cells }}$ of cells, the number $N b_{\text {nodes }}$ of nodes, the number $N b_{F r a c F}$ of fracture faces, the number of d.o.f. $\operatorname{Card}\left(\mathcal{V} \cup \mathcal{M} \cup \mathcal{F}_{\Gamma}\right)$ of the scheme (with two unknowns per d.o.f.), and the number of d.o.f. $\operatorname{Card}\left(\mathcal{V} / \mathcal{V}_{D} \cup \mathcal{F}_{\Gamma}\right)$ of the linear system (with two unknowns per d.o.f.) after elimination without fill-in of the cells and Dirichlet nodes, where $\mathcal{V}_{D}$ is the set of Dirichlet nodes at the boundary of $\Omega$. It also specifies for each mesh the cubic root of the mean volume of all cells at the matrix fracture 
interface, denoted by $\rho_{i}$, and defined by

$$
\varrho_{i}=\left(\frac{1}{\operatorname{Card}\{K \in \mathcal{M} \mid K \cap \Gamma \neq \emptyset\}} \sum_{K \in \mathcal{M} \mid K \cap \Gamma \neq \emptyset}|K|\right)^{1 / 3} .
$$
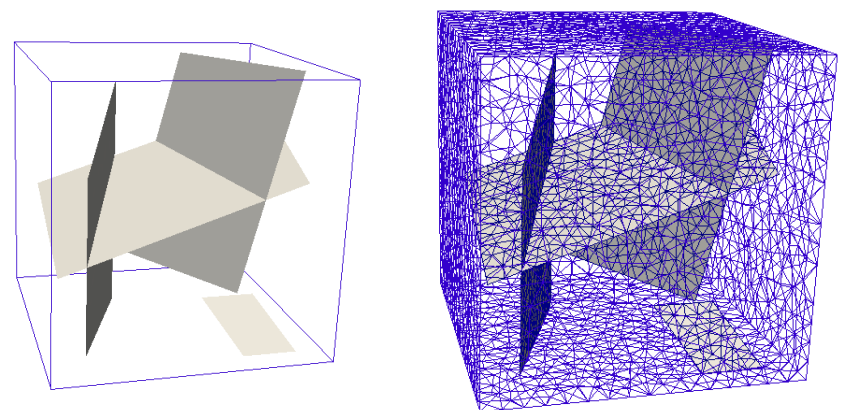

Figure 12: Geometry of the basin, fracture network, and coarsest three-dimensional Delaunay mesh $i_{\text {mesh }}=1$.

\begin{tabular}{|c|c|c|c|c|c|c|c|}
\hline$i_{\text {mesh }}$ & $N b_{\text {cells }}$ & $N b_{\text {nodes }}$ & $N b_{F r a c F}$ & $\varrho_{i}$ & scheme d.o.f. & linear system d.o.f. & $\theta_{\mathcal{T}}$ \\
\hline 1 & 47670 & 8348 & 1678 & 2.42 & 57696 & 9278 & 209 \\
\hline 2 & 253945 & 41043 & 6655 & 1.23 & 301643 & 46283 & 86 \\
\hline 3 & 837487 & 132778 & 16497 & 0.78 & 986762 & 147148 & 142 \\
\hline 4 & 3076262 & 483786 & 42966 & 0.48 & 3603014 & 523453 & 200 \\
\hline
\end{tabular}

Table 3: For each mesh: number $N b_{\text {cells }}$ of mesh cells, number $N b_{\text {nodes }}$ of nodes, number $N b_{F r a c F}$ of fracture faces, scheme and linear system number of d.o.f. (with 2 unknowns per d.o.f), cubic root of the average cell volume at the matrix fracture interface $\varrho_{i}$, and shape regularity $\theta_{\mathcal{T}}$.

We suppose again a high contrast between the permeability of the matrix $\Lambda_{m}=\lambda_{m}$ Id and the permeability of the fractures $\Lambda_{f}=\lambda_{f}$ Id with $\lambda_{m}=10^{-17} \mathrm{~m}^{2}, \lambda_{f}=10^{-11} \mathrm{~m}^{2}$. The porosities, the phase densities, the mobilities and the capillary pressures are the same than in the previous test case. The initial and boundary conditions are also the same except that the oil phase is injected at the full bottom side of the domain. The simulation is run over a period of 10 years with an initial time step of 0.2 days, and a maximum time step fixed to 5 days, except on mesh 4 for which a smaller maximum time step of 2.5 days is used. All the numerical tests have been performed on the Cicada Cluster located at the University Nice Sophia-Antipolis and which includes 1152 nodes equipped with two eight-core Intel(R) E5-2670 processors. Figure 13 exhibits the oil saturation obtained on the coarsest mesh $i_{\text {mesh }}=1$ at final simulation time. We observe that the oil phase injected at the bottom side in the domain initially saturated with water, quickly rises by gravity along the faults and slowly penetrate in the matrix. 


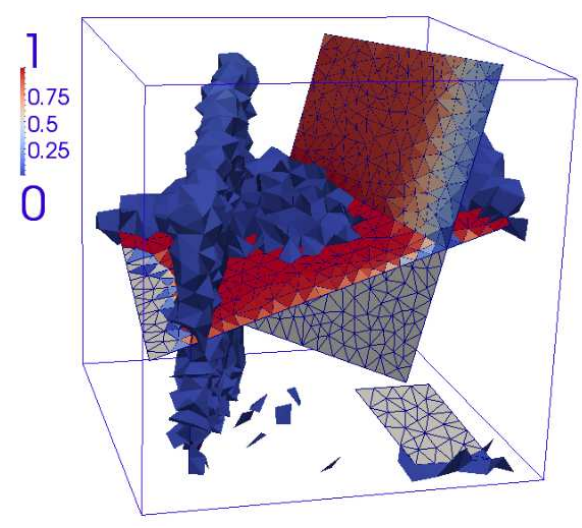

Figure 13: Discrete solution obtained by the VAG-1 scheme with the first mesh $i_{\text {mesh }}=1$ at final simulation time: oil saturation in the fracture network and in the matrix using the lower threshold in the matrix equal to 0.001 .

Figure 14 compares the convergence of the oil saturation on the family of refined meshes for the two choices of the volume fractions VAG-1 and VAG-2. It is clear, as in the 2D test case, for such high ratio of the fracture and matrix permeabilities, that VAG-1 provides a much better convergence than VAG-2 since it does not mix porous volumes from the matrix and the fracture network. It illustrates again the advantage of the VAG scheme compared with CVFE discretizations which cannot avoid such mixing of porous volumes. Table 4.3 presents the numerical behavior of the simulations for both choices of the distribution of the volumes and for the family of meshes. The results obtained demonstrate the good robustness and scalability of the proposed numerical scheme both in terms of Newton convergence, linear solver convergence and CPU time. As for the $2 \mathrm{D}$ network test case, the robustness of the nonlinear solver is slightly better for VAG-2 due to the larger control volumes at the matrix fracture interfaces for VAG-2 than for VGA-1.
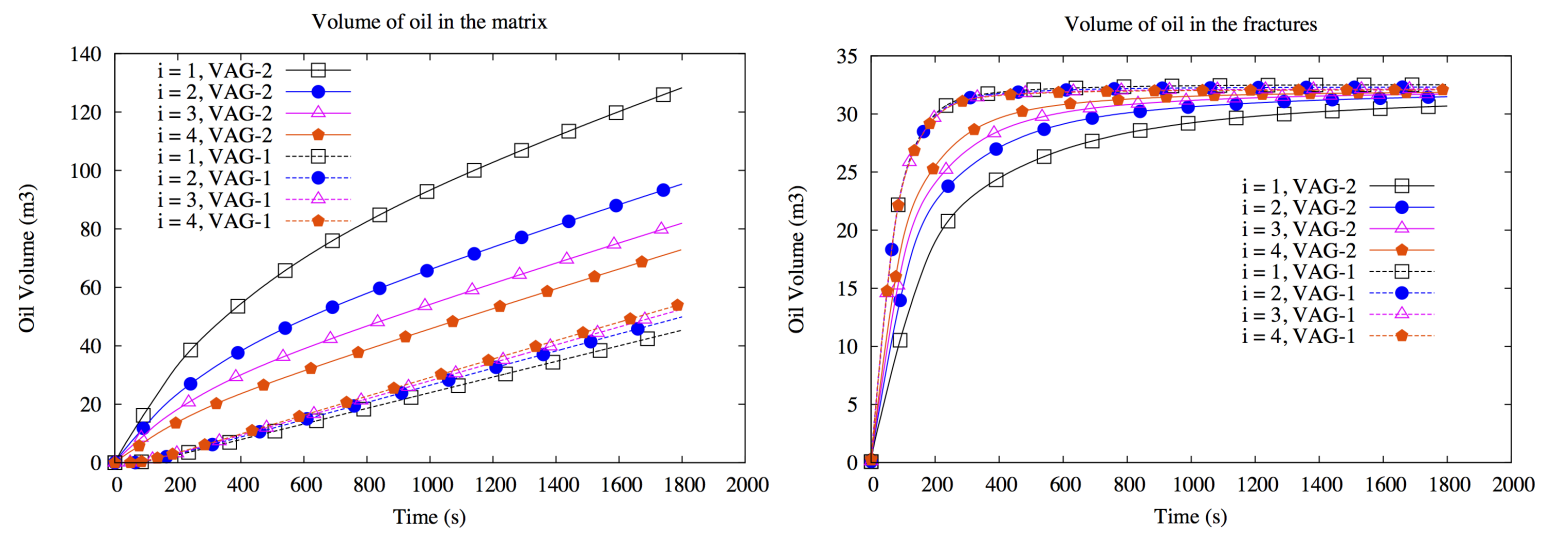

Figure 14: Volumes of oil in the fracture and in the matrix function of time for the family of meshes $i_{m e s h}=1, . ., 4$, and for both choices VAG-1 and VAG-2 of the volume distribution. 


\begin{tabular}{|c|c|c|c|c|c|c|}
\hline Volumes & $i_{\text {mesh }}$ & $N_{\Delta t}$ & $N_{\text {Chop }}$ & $N_{\text {Newton }}$ & $N_{\text {GMRes }}$ & CPU (s) \\
\hline VAG-1 & 1 & 384 & 6 & 2.20 & 10.05 & 588 \\
\hline VAG-1 & 2 & 390 & 10 & 3.08 & 15.11 & 5898 \\
\hline VAG-1 & 3 & 415 & 21 & 4.02 & 15.93 & 31806 \\
\hline VAG-1 & 4 & 784 & 30 & 3.37 & 16.75 & 209485 \\
\hline \hline VAG-2 & 1 & 373 & 0 & 1.87 & 6.94 & 482 \\
\hline VAG-2 & 2 & 373 & 0 & 2.42 & 13.05 & 4452 \\
\hline VAG-2 & 3 & 375 & 1 & 3.02 & 14.56 & 21645 \\
\hline VAG-2 & 4 & 747 & 13 & 2.92 & 16.55 & 172946 \\
\hline
\end{tabular}

Table 4: For each choice VAG-1 and VAG-2 of the volume distribution and for each mesh $i_{m e s h}=$ $1, . ., 4$ : number $N_{\Delta t}$ of successful time steps, number $N_{C h o p}$ of time step chops, number $N_{N e w t o n}$ of Newton iterations per successful time step, number $N_{G M R e s}$ of GMRes iterations by Newton iteration, CPU time in seconds.

\section{Conclusion}

This paper has introduced the VAG discretization of hybrid dimensional two-phase Darcy flows modelling discrete fracture networks with mass exchange between the matrix and the fractures. Our discretization takes into account general polyhedral meshes, general discrete fracture networks, the anisotropy of the matrix and of the fracture permeability fields, and discontinuous rocktypes.

Compared with CVFE approaches, the numerical tests clearly exhibit that the VAG scheme has the advantage to avoid the mixing of the fracture and matrix rocktypes at the interfaces between the matrix and the fractures, while keeping the low cost of a nodal discretization on unstructured meshes.

The convergence of the scheme to a weak solution of the model has been proved for arbitrary choices of the volumes at the nodal unknowns assuming the non degeneracy of the relative permeabilities and a network of planar fractures. To our knowledge, this is the first convergence result for this type of hybrid dimensional two-phase Darcy flow model, and it also provides an existence result for such models.

\section{Appendix}

This appendix presents two technical lemmas used in the convergence proof. The following lemma states the weak compactness of bounded sequences in $L^{2}\left(0, T ; V^{0}\right)$.

Lemma 5.1 Let $\left(v^{(m)}\right)_{m \in \mathbb{N}}$ be a sequence of functions in $L^{2}\left(0, T ; V^{0}\right)$ such that $\left\|v^{(m)}\right\|_{L^{2}(0, T ; V)} \leq C$ for some positive $C$. Then, there exists $v \in L^{2}\left(0, T ; V^{0}\right)$ such that up to the same subsequence

$$
v^{(m)} \rightarrow v \text { in } L^{2}(\Omega \times(0, T)) \text { and } \gamma v^{(m)} \rightarrow \gamma v \text { in } L^{2}(\Gamma \times(0, T)) ;
$$

moreover

$$
\nabla v^{(m)} \rightarrow \nabla v \quad \text { in } L^{2}(\Omega \times(0, T))^{d} \quad \text { and } \quad \nabla \gamma v^{(m)} \rightarrow \nabla \gamma v \quad \text { in } \quad L^{2}(\Gamma \times(0, T))^{d-1} .
$$

Proof: First, let us recall that the normal trace operator $\gamma_{\mathbf{n}}: H_{\text {div }}(\Omega) \rightarrow H^{-1 / 2}(\partial \Omega)$ is surjective. Indeed, for any $l \in H^{-1 / 2}(\partial \Omega)$ we consider the unique weak solution $u \in H^{1}(\Omega)$ of $-\Delta u+u=0$ on $\Omega$ with $\nabla u \cdot \mathbf{n}=l$ on $\partial \Omega$ defined by $\langle u, v\rangle_{H_{1}(\Omega)}=\left\langle l, \gamma_{\partial \Omega} v\right\rangle_{H^{-1 / 2}(\partial \Omega), H^{1 / 2}(\partial \Omega)}$ for all $v \in H^{1}(\Omega)$, where $\gamma_{\partial \Omega}$ is the trace operator from $H^{1}(\Omega)$ to $H^{1 / 2}(\partial \Omega)$. Hence the function $\mathrm{G}:=\nabla u$ is in $H_{\text {div }}(\Omega)$ and satisfies $\gamma_{\mathbf{n}} \mathrm{G}=l$.

Next, from the Poincare inequality and the continuity of the trace operators $\gamma_{i}$, the sequence $v^{(m)}$ is bounded in $L^{2}\left(0, T ; H^{1}(\Omega)\right)$ and the sequences $v_{i}^{(m)}=\gamma_{i} v^{(m)}$ are bounded in $L^{2}\left(0, T ; H^{1}\left(\Gamma_{i}\right)\right)$ for all 
$i \in I$. Hence, it is classical to show that there exist $v \in L^{2}\left(0, T ; H_{0}^{1}(\Omega)\right)$ and $v_{i} \in L^{2}\left(0, T ; H_{\Sigma_{i, 0}}^{1}\left(\Gamma_{i}\right)\right)$ such that up to the same subsequence:

$$
\left\{\begin{array}{cl}
v^{(m)}-v & \text { in } L^{2}(\Omega \times(0, T)), \\
\nabla v^{(m)}-\nabla v & \text { in } L^{2}(\Omega \times(0, T))^{d}, \\
v_{i}^{(m)}-v_{i} & \text { in } L^{2}\left(\Gamma_{i} \times(0, T)\right), \\
\nabla v_{i}^{(m)}-\nabla v_{i} & \text { in } L^{2}\left(\Gamma_{i} \times(0, T)\right)^{d-1},
\end{array}\right.
$$

for all $i \in I$, where $H_{\Sigma_{i, 0}}^{1}\left(\Gamma_{i}\right)$ is the subspace of $H^{1}\left(\Gamma_{i}\right.$ with vanishing trace on $\Sigma_{i, 0}$. It remains to show that $v \in L^{2}\left(0, T ; V^{0}\right)$ with $(\gamma v)_{i}=v_{i}$. Let us first show that $\gamma_{i} v=v_{i}$. We extend the fracture $\Gamma_{i}$ in the direction of the plane $\mathcal{P}_{i}$ in order to reach $\partial \Omega$ and to decompose the domain $\Omega$ into two subdomains, say $\Omega_{i}$ and $\Omega_{i^{\prime}}$. Now let us introduce $r_{i} \in L^{2}\left(\Gamma_{i}\right)$ and a function $l \in L^{2}\left(\partial \Omega_{i}\right)$ which is defined by

$$
l= \begin{cases}r_{i} & \text { on } \Gamma_{i} \\ 0 & \text { otherwise }\end{cases}
$$

Thus, since $l \in L^{2}\left(\partial \Omega_{i}\right) \subset H^{-1 / 2}\left(\partial \Omega_{i}\right)$ and, thanks to the surjectivity of the normal trace operator recalled above, there exists $\mathbf{q}_{i} \in H_{\text {div }}\left(\Omega_{i}\right)$ such that $\mathbf{q}_{i} \cdot \mathbf{n}_{i}=l$. Then, passing to the limit in the equality

$$
\int_{0}^{T} \int_{\Omega_{i}}\left(\mathbf{q}_{i}(\mathbf{x}) \cdot \nabla v^{(m)}(\mathbf{x}, t)+v^{(m)}(\mathbf{x}, t) \operatorname{div} \mathbf{q}_{i}(\mathbf{x})\right) \varphi(t) d \mathbf{x} d t=\int_{0}^{T} \int_{\Gamma_{i}} \varphi(t) r_{i}(\mathbf{x}) v_{i}^{(m)}(\mathbf{x}, t) d \tau(\mathbf{x}) d t,
$$

yields

$$
\int_{0}^{T} \int_{\Gamma_{i}} \varphi(t) r_{i}(\mathbf{x})\left(v_{i}(\mathbf{x}, t)-\gamma_{i} v(\mathbf{x}, t)\right) d \tau(\mathbf{x}) d t=0,
$$

for all $r_{i} \in L^{2}\left(\Gamma_{i}\right)$ and $\varphi \in L^{2}(0, T)$, hence $v_{i}=\gamma_{i} v$. Next, let us introduce the following trace operators

$$
\gamma_{i, j}: H^{1}\left(\Gamma_{i}\right) \rightarrow L^{2}\left(\Sigma_{i, j}\right)
$$

for each $(i, j) \in I_{\Sigma}^{2}$ where $I_{\Sigma}^{2}$ is the subset of $I \times I$ such that $\Sigma_{i, j}$ has a non zero $d-2$ dimensional Lebesgue measure. We will prove that $\gamma_{i, j} v_{i}=\gamma_{j, i} v_{j}$ for all $(i, j) \in I_{\Sigma}^{2}$. Let us introduce $r_{i, j}=-r_{j, i} \in$ $L^{2}\left(\Sigma_{i, j}\right)$ and the function $l_{i}$ and $l_{j}$ defined by

$$
l_{i}= \begin{cases}r_{i, j} & \text { on } \Sigma_{i, j} \\ 0 & \text { on } \partial \Gamma_{i} \backslash \Sigma_{i, j}\end{cases}
$$

and

$$
l_{j}= \begin{cases}r_{j, i} & \text { on } \Sigma_{i, j} \\ 0 & \text { on } \partial \Gamma_{j} \backslash \Sigma_{i, j}\end{cases}
$$

Thus, thanks again to the surjectivity of the normal trace operator, there exist $\mathbf{q}_{i} \in H_{\text {div }}\left(\Gamma_{i}\right)$ and $\mathbf{q}_{j} \in H_{\text {div }}\left(\Gamma_{j}\right)$ such that $\mathbf{q}_{i} \cdot \mathbf{n}_{i}=l_{i}$ and $\mathbf{q}_{j} \cdot \mathbf{n}_{j}=l_{j}$. Then, passing to the limit in the equality

$$
\int_{0}^{T} \varphi(t) \int_{\Gamma_{i}} \nabla v_{i}^{(m)} \cdot \mathbf{q}_{i}+v_{i}^{(m)} \operatorname{div}\left(\mathbf{q}_{i}\right) d \tau(\mathbf{x}) d t+\int_{0}^{T} \varphi(t) \int_{\Gamma_{j}} \nabla v_{j}^{(m)} \cdot \mathbf{q}_{j}+v_{j}^{(m)} \operatorname{div}\left(\mathbf{q}_{j}\right) d \tau(\mathbf{x}) d t=0,
$$

we obtain that

$$
\int_{0}^{T} \int_{\Sigma_{i, j}} \varphi(t) r_{i, j}(\mathbf{x})\left(\gamma_{i, j} v_{i}(\mathbf{x}, t)-\gamma_{j, i} v_{j}(\mathbf{x}, t)\right) d l(\mathbf{x}) d t=0,
$$

for all $r_{i, j} \in L^{2}\left(\Sigma_{i, j}\right), \varphi \in L^{2}(0, T)$ which implies that $\gamma_{i, j} v_{i}=\gamma_{j, i} v_{j}$, and hence that $v \in L^{2}\left(0, T ; V^{0}\right)$.

The following Lemma states an estimate on the space translates for bounded sequences in $L^{2}\left(0, T ; H_{0}^{1}(\Omega)\right)$ or in $L^{2}\left(0, T ; H_{0}^{1}\left(\Gamma_{i}\right)\right)$. 
Lemma 5.2 Let $d \in \mathbb{N}^{*}, t>0$ and let $\Upsilon$ be an open polyhedral $(d=3)$ or polygonal $(d=2)$ domain in $\mathbb{R}^{d}$. Let $\left(u^{(m)}\right)_{m \in \mathbb{N}}$ be a sequence of functions from $L^{2}\left(0, T ; H_{0}^{1}(\Upsilon)\right)$ such that $\left\|u^{(m)}\right\|_{L^{2}\left(0, T ; H^{1}(\Upsilon)\right)}<C$ for some positive $C$. Then, extending the functions $u^{(m)}$ by 0 on $\left(\mathbb{R}^{d} \backslash \Upsilon\right) \times(0, T)$, one has

$$
\sup _{m \in \mathbb{N}}\left\|u^{(m)}(\cdot, \cdot+\xi)-u^{(m)}\right\|_{L^{2}\left(\mathbb{R}^{d} \times(0, T)\right)}^{2} \rightarrow 0 \text { as }|\xi| \rightarrow 0 \text {. }
$$

Proof : It is sufficient to notice that for all $\varphi \in C_{\infty}^{c}\left(\mathbb{R}^{d} \times(0, T)\right)$ one has

$$
\|\varphi(\cdot+\xi)-\varphi\|_{L^{2}\left(\mathbb{R}^{d} \times(0, T)\right)}=|\xi|\|\nabla \varphi\|_{\left(L^{2}\left(\mathbb{R}^{d} \times(0, T)\right)\right)^{d}} .
$$

Therefore the result is deduced form the density of the set $C_{\infty}^{c}\left(\mathbb{R}^{d} \times(0, T)\right)$ in $L^{2}\left(0, T ; H^{1}\left(\mathbb{R}^{d}\right)\right)$ and from the fact that $u^{(m)} \in L^{2}\left(0, T ; H^{1}\left(\mathbb{R}^{d}\right)\right)$.

\section{Acknowledgements}

The authors would like to thank GDFSuez EP and Storengy for partially supporting this work.

\section{References}

[1] C. Alboin, J. Jaffré, J. Roberts, C. Serres, Modeling fractures as interfaces for flow and transport in porous media. In: Chen, Ewing, editors. Fluid flow and transport in porous media, vol. 295. American Mathematical Society, pp. 13-24, 2002.

[2] O. Angelini, K. Brenner, D. Hilhorst, A finite volume method on general meshes for a degenerate parabolic convection-reaction-diffusion equation, Numer. Math., vol. 123, issue 2, pp. 219-257, 2013.

[3] P. Angot, F. Boyer, F. Hubert, Asymptotic and numerical modelling of flows in fractured porous media, M2AN, Volume 23, pp.239-275, 2009.

[4] S. N. Antontsev, A. V. Kazhikhov, and V. N. Monakhov. Boundary value problems in mechanics of nonhomogeneous fluids, volume 22 of Studies in Mathematics and its Applications. NorthHolland Publishing Co., Amsterdam, 1990. Translated from Russian.

[5] B.R. Baliga and S.V. Patankar SV, A control volume finite-element method for two dimensional fluid flow and heat transfer. Numerical Heat Transfer, vol. 6, pp. 245-261, 1983.

[6] K. Brenner, R. Masson, Convergence of a Vertex centred Discretization of Two-Phase Darcy flows on General Meshes, Int. Journal of Finite Volume Methods, june 2013.

[7] K. Brenner, C. Cancès, and D. Hilhorst, Finite volume approximation for an immiscible two-phase flow in porous media with discontinuous capillary pressure, Computational Geosciences, 2013.

[8] C. Cancès, Finite volume scheme for two-phase flows in heterogeneous porous media involving capillary pressure discontinuities, M2AN, Mathematical Modelling and Numerical Analysis, 43,5, pp. 973-1001, 2009.

[9] Cancès, Clément and Pierre, Michel, An existence result for multidimensional immiscible twophase flows with discontinuous capillary pressure field, SIAM J. Math. Anal., 44,2, pp. 966-992, 2012 .

[10] G. Chavent and J. Jaffré. Mathematical Models and Finite Elements for Reservoir Simulation, volume 17. North-Holland, Amsterdam, stud. math. appl. edition, 1986.

[11] C. J. van Duijn, J. Molenaar, and M. J. de Neef, The effect of capillary forces on immiscible two-phase flows in heterogeneous porous media, Transp. Porous Media, 21, pp. 71- 93, 1995. 
[12] R. Eymard, R. Herbin, and A. Michel. Mathematical study of a petroleum-engineering scheme. M2AN Math. Model. Numer. Anal., 37(6) pp. 937-972, 2003.

[13] R. Eymard, C. Guichard, and R. Herbin, Small-stencil 3D schemes for diffusive flows in porous media. ESAIM: Mathematical Modelling and Numerical Analysis, 46, pp. 265-290, 2010.

[14] R. Eymard, C. Guichard, R. Herbin, and R. Masson, Vertex centred Discretization of Two-Phase Darcy flows on General Meshes, ESAIM Proceedings, volume 35, pp. 59-78, 2012.

[15] R. Eymard, R. Herbin, C. Guichard, R. Masson, Vertex Centred discretization of compositional Multiphase Darcy flows on general meshes. Comp. Geosciences, vol. 16, issue 4, pp. 987-1005, 2012 .

[16] R. Eymard, P. Féron, T. Gallouët, R. Herbin, C. Guichard. Gradient schemes for the Stefan problem. IJFV - International Journal on Finite Volumes, june 2013.

[17] R. Eymard, C. Guichard, R. Herbin, R. Masson, Gradient schemes for two-phase flow in heterogeneous porous media and Richards equation, article first published online, ZAMM - Journal of Applied Mathematics and Mechanics, 2013. doi: 10.1002/zamm.201200206

[18] E. Flauraud, F. Nataf, I. Faille, R. Masson, Domain Decomposition for an asymptotic geological fault modeling, Comptes Rendus à l'académie des Sciences, Mécanique, 331, pp 849-855, 2003.

[19] Luca Formaggia, Alessio Fumagalli, Anna Scotti, Paolo Ruffo A reduced model for Darcy's problem in networks of fractures, to appear in ESAIM Mathematical Modelling and Numerical Analysis, 2014.

[20] H. Haegland, I. Aavatsmark, C. Guichard, R. Masson, R. Kaufmann, Comparison of a Finite Element Method and a Finite Volume Method for Flow on General Grids in 3D, In the proceedings of ECMOR XIII, Biarritz, september 2012.

[21] J. Hoteit, A. Firoozabadi, An efficient numerical model for incompressible two-phase flow in fracture media, Advances in Water Resources, 31, pp. 891-905, 2008.

[22] R. Huber and R. Helmig, Node-centred finite volume discretizations for the numerical simulation of multi-phase flow in heterogeneous porous media, Computational Geosciences, 4, pp. 141-164, 2000 .

[23] J. Jaffré, M. Mnejja, J.E. Roberts, A discrete fracture model for two-phase flow with matrixfracture interaction, Procedia Computer Science 4, pp. 967-973, 2011.

[24] M. Karimi-Fard, L.J. Durlovski, K. Aziz, An efficient discrete-fracture model applicable for general-purpose reservoir simulators, SPE journal, june 2004.

[25] Lacroix, S. and Vassilevski, Y. V. and Wheeler, M. F.: Decoupling preconditioners in the Implicit Parallel Accurate Reservoir Simulator (IPARS). Numerical Linear Algebra with Applications, 8, pp. 537-549, 2001.

[26] V. Martin, J. Jaffré, J. E. Roberts, Modeling fractures and barriers as interfaces for flow in porous media, SIAM J. Sci. Comput. 26 (5), pp. 1667-1691, 2005.

[27] A. Michel, A finite volume scheme for two-phase immiscible flow in porous media, SIAM J. Numer. Anal., 41(4), pp. 1301-1317, 2003.

[28] J. Monteagudu, A. Firoozabadi, Control-volume model for simulation of water injection in fractured media: incorporating matrix heterogeneity and reservoir wettability effects, SPE J. 12, pp. 355-366, 2007. 
[29] V. Reichenberger, H. Jakobs, P. Bastian, R. Helmig, A mixed-dimensional finite volume method for multiphase flow in fractured porous media, Adv. Water Resources 29 (7), pp. 1020-1036, 2006.

[30] Scheichl, R. and Masson, R. and Wendebourg, J.: Decoupling and block preconditioning for sedimentary basin simulations. Computational Geosciences, 7, pp. 295-318, 2003.

[31] X. Tunc, I. Faille, T. Gallouet, M.C. Cacas, P. Havé, A model for conductive faults with non matching grids, Comp. Geosciences, 16, pp. 277-296, 2012.

[32] http://tetgen.org 